2 The mechanism of $\mathrm{Mg}$ diffusion in forsterite and the controls on its anisotropy

3 Joshua M. R. Muir*1,2, Feiwu Zhang, and Andrew M. Walker2

4 1) Institute of Geochemistry, Chinese Academy of Sciences, 99 West Lincheng Road, Guiyang, Guizhou 550081, China

5 2) School of Earth and Environment, University of Leeds, LS2 9JT, United Kingdom

7 *Corresponding author: j.m.r.muir@mail.gyig.ac.cn, a.walker@leeds.ac.uk, zhangfeiwu@mail.gyig.ac.cn

10 This is an EarthArvix.org preprint which has been peer reviewed but not yet accepted. It has been 11 submitted to Physics of the Earth and Planetary Interiors for further review. Subsequent versions may have some changes in content. Please feel free to contact any of the authors, we welcome 13 feedback. 


\section{The mechanism of $\mathrm{Mg}$ diffusion in forsterite and the controls on its anisotropy}

Joshua M. R. Muir ${ }^{* 1,2}$, Zhang Feiwu ${ }^{1}$ and Andrew M. Walker ${ }^{2,3}$

1) Institute of Geochemistry, Chinese Academy of Sciences, 99 West Lincheng Road, Guiyang, Guizhou 550081, China

2) School of Earth and Environment, University of Leeds, LS2 9JT, United Kingdom

3) Now at: Department of Earth Sciences, University of Oxford, South Parks Road, Oxford OX1 3AN, United Kingdom

*Corresponding author: j.m.r.muir@mail.gyig.ac.cn, andrew.walker@earth.ox.ac.uk, zhangfeiwu@mail.gyig.ac.cn

1

22

\section{Abstract}

Mg diffusion is important for explaining many rheological properties in forsterite but its mechanism is unknown. Without knowing a mechanism the effect of variables such as pressure are hard to constrain. In this study we used Density Functional Theory (DFT) to calculate the diffusivity of Mg vacancies and interstitials in forsterite and thus the diffusion rate of $\mathrm{Mg}$ in forsterite. We predict vacancy diffusion to be highly anisotropic with considerably faster diffusion in the [001] direction while interstitial diffusion is predicted to be more isotropic. Thus we predict that a combination of interstitial and vacancy diffusion is required to reproduce experimentally derived anisotropies. Interstitial diffusion is predicted to be highly pressure dependant such that with increasing pressure the anisotropy of $\mathrm{Mg}$ diffusion decreases while temperature has little effect on this anisotropy. Substances like Fe and water likely cause increases in $\mathrm{Mg}$ diffusion rate through the creation of extrinsic Mg vacancies and we predict that without modifications to the inherent mobility of $\mathrm{Mg}$ vacancies these cause small increases to diffusional anisotropy at 1300 and $1600 \mathrm{~K}$ but very large increases at $1000 \mathrm{~K}$.

Keywords: Forsterite; Mg Diffusion; DFT

Word Count: 7504 


\section{Introduction}

Diffusion of cations occupying the octahedral metal sites in olivine controls processes that are active in the Earth's crust and upper mantle, and which underpin a range of geophysical and geochemical techniques. In the upper mantle, where olivine with composition close to $\left(\mathrm{Mg}_{0.9}, \mathrm{Fe}_{0.1}\right)_{2} \mathrm{SiO}_{4}$ is the dominant phase, the diffusivity of $\mathrm{Mg}$ is important in understanding electrical conductivity (Fei et al., 2018, Yoshino et al., 2009, Yoshino et al., 2017, Schock et al., 1989) and could influence deformation even though $\mathrm{Mg}$ is a rapidly diffusing species as argued in Jaoul (1990). Anisotropic Mg diffusion could be an important factor in explaining the anisotropic conduction seen in high conductivity layers underneath young oceanic plates (Fei et al., 2018) and, if Mg diffusion is important in forming olivine textures, could also help explain the variety of textures that are formed by olivine under different conditions (Karato et al., 2008). Mg-Fe interdiffusion occurring in zoned phenocrysts from volcanic products is increasingly used as a petrological tool (diffusion chronometry) to understand the timescales of pre-eruptive processes operating in the days and weeks prior to eruption (e.g. Hartley et al. 2016 and Pankhurst et al. 2018). On a longer timescale diffusion-controlled exchange between $\mathrm{Mg}$ and Fe in olivine and spinel can be used to infer the post-crystallisation thermal history of ultramafic igneous bodies (Ozawa, 1984). Diffusion can also lead to magnesium and iron isotope fractionation (Teng et al., 2011).

Our understanding and ability to model all of these processes relies on accurate determination of the Mg self-diffusion and Fe-Mg interdiffusion coefficients in olivine and thus this has been the focus of a range of experimental and computational studies reviewed by Chakraborty (2010). However, details of the atomic scale basis of $\mathrm{Mg}$ self and inter-diffusion in olivine have thus far eluded a full atomistic explanation and this limits our ability to confidently make use of this data under the wide range of conditions where diffusion is important. In this work we shall study the atomistic mechanisms of $\mathrm{Mg}$ self-diffusion as the more straightforward of these two processes.

\section{Experimental measures of $\mathrm{Mg}$ diffusion}


Previous experimental studies have identified several key features of $\mathrm{Mg}$ self-diffusion as well as questions that remain unanswered. Despite early uncertainty, it is clear that magnesium self-diffusion is faster than the self-diffusion of oxygen or silicon (for a review of this history see Chakraborty (2010)). Diffusion can be described by a basic equation $D^{s d}=D_{0} \exp \left(-\frac{E_{a c t}}{k_{B} T}\right)$ where $D^{\text {sd }}$ is the rate of selfdiffusion, $D_{0}$ is a preexponential factor, $E_{a c t}$ is the activation energy, $k_{B}$ is the Boltzmann constant and $T$ is the temperature. $D_{0}$ and $E_{a c t}$ can then be treated as fitting factors for experiments run at different temperatures. Recent experimental estimates of $E_{\text {act }}$ and $D_{0}$ for $M g$ tracer diffusion in forsterite are $9.6 \times 10^{-4} \mathrm{~m}^{2} / \mathrm{s}$ and $4.15 \pm 0.17 \mathrm{eV}$ (Chakraborty et al., 1994) or $4.0 \times 10^{-9} \mathrm{~m}^{2} / \mathrm{s}$ or $2.59 \pm 0.31 \mathrm{eV}$ (Fei et al., 2018). The diffusion rate $D_{s d}$ at $1300 \mathrm{~K}$ and $0 \mathrm{GPa}$ is $3.6 \times 10^{-19} \mathrm{~m}^{2} / \mathrm{s}$ in Fei et al. (2018a) or $2-6 \times 10^{19} \mathrm{~m}^{2} / \mathrm{s}$ in Chakraborty et al. 1994. More generally there is broad agreement as to the Mg diffusion parameters in anhydrous forsterite though there is around half an order of magnitude discrepancy between different experimental predictions, some of which are plotted in Figure 5 (Andersson et al., 1989, Chakraborty et al., 1994, Fei et al., 2018, Jollands et al., 2020, Morioka, 1981) .

In detail, magnesium self-diffusivity is found to be mildly sensitive to pressure, to be anisotropic and to depend on the chemistry of the olivine crystal. Diffusion along [001] has been found to be faster than diffusion along [100], which is faster than diffusion along [010] (Chakraborty et al., 1994) though other studies have found diffusion along [010] to be faster than diffusion along [100] (Andersson, 1987, Jollands et al., 2020). There are some differences in experimental activation volumes 1-3.5 $\mathrm{cm}^{3} / \mathrm{mol}$ (Chakraborty et al., 1994) or $4.0-4.6 \mathrm{~cm}^{3} / \mathrm{mol}$ (Fei et al., 2018) but in all cases these are small and so pressure has little effect on diffusion rates. These activation volumes come from data solely in the [001] direction which is important because they do not reflect all processes that occur in the crystal as shall be explored in the text.

Although these experiments provide the critical data needed to model diffusion-controlled processes in olivine, several aspects of $\mathrm{Mg}$ diffusion remain enigmatic and some parameters have not been fully established. For example, the reason for the anisotropy of diffusion is not clear and the effect of 
pressure or other elements on this anisotropy has not yet been determined. An argument has been made that diffusional anisotropy is related to Mg hopping distance (Brodholt, 1997) but in a highly anisotropic crystal like forsterite the difficulty of $\mathrm{Mg}$ hopping is unlikely to be a simple function of distance. By using ab-initio calculations we can explore atomistic mechanisms by which these processes could occur.

\section{Crystal structure of olivine}

In order to understand diffusion in olivine it is first necessary to consider the crystal structure, how this permits point defect mobility and the chemistry that controls the defect concentration. This will allow us to formulate possible atomistic mechanisms by which diffusion can occur. Olivine's distorted hexagonal close packed oxygen sublattice contains two distinct octahedrally co-ordinated M sites: M1, on an inversion centre, and the less symmetric $\mathrm{M} 2$, which sits on a mirror plane. $\mathrm{M} 1$ sites share edges and form continuous chains along [001] while M2 sites are isolated from each other (sharing an edge with an $\mathrm{M} 1$ site and corners with other $\mathrm{M} 1$ and $\mathrm{M} 2$ sites). This structural anisotropy hits at a possible reason for the directional dependence of $\mathrm{Mg}$ diffusion in forsterite: vacancy mobility along chains of M1 sites could be high compared to more tortuous pathways between M1 and M2 sites. There are also two normally unoccupied octahedral sites in the olivine structure. Each is located half way between two occupied $M$ sites (and shares faces with them) along [100]. We call the unoccupied octahedral site midway between two M1 sites $I 1$, and the unoccupied octahedral site midway between two M2 sites 12.

\section{Theoretical Studies of $\mathrm{Mg}$ defects and their diffusion in Forsterite}

Using this knowledge of forsterite crystal structure previous work has attempted to address the structure and mobility of point defects in forsterite. This largely consists of two parts, first identifying the points defects of forsterite and then their mobility.

Simulations using interatomic potentials (Wright and Catlow, 1994, Walker et al., 2009, Jaoul et al., 1995, Bejina et al., 2009), density functional theory (Brodholt, 1997) and QM/MM embedded clusters (Walker et al., 2009, Braithwaite et al., 2003) suggest that the most important defects are Mg 
vacancies which are most stable on M1 rather than M2 sites and octahedrally coordinated Mg interstitials which form a split-interstitial structure (two magnesium ions in tetrahedral coordination located on opposite sides of the M1 site) is stable (Walker et al., 2009). The mobility of some of these defects has been studied using interatomic potentials (Bejina et al., 2009, Jaoul et al., 1995, Walker et al., 2009) where it was found that $\mathrm{Mg}$ vacancies are more mobile than Mg interstitials (Walker et al., 2009), that pressure has a limited effect on mobility along the M1 chain as was found in experiments (Jaoul et al., 1995, Bejina et al., 2009) and that vacancies overwhelmingly diffuse along the [001] M1 chain (Bejina et al., 2009). These studies have neglected important effects. First, interatomic potentials often behave poorly in unusual geometries and these are often formed during diffusion. Second, these studies consider only activation energies and not the time taken for diffusing point defects to overcome these barriers. And third, they do not convert their diffusion pathways into a macroscopic diffusion model and thus calculate rates of diffusion.

Thus there exists no detailed exploration of $\mathrm{Mg}$ diffusion in forsterite using electronic structure methods. In the following we make use of atomic scale simulation to understand the atomic scale mechanism of $\mathrm{Mg}$ diffusion in forsterite, determine the absolute diffusivity as a function of direction and how this is altered by pressure and how extrinsic defects could affect this picture.

\section{Methods}

Compared to the timescale accessible to direct atomic scale simulation using molecular dynamics, point defect diffusion in minerals is usually slow. Methods available to simulate diffusion thus seek to describe diffusion by repeated rare events which can be studied in detail, and then combined in order to describe diffusion on a meaningful timescale. The rare events are typically hops of point defects between adjacent sites. For example, one of a number of atoms could migrate into a vacancy, effectively moving the vacancy and permitting diffusion via a vacancy mechanism, or an interstitial atom could move into one of a number of different interstitial sites, permitting diffusion via an interstitial mechanism. Repeated occurrences of these hops leads to a random walk of the defect and 
bulk self-diffusion (Tilley, 1987). Our approach to simulating Mg diffusion in forsterite thus follows three steps. First, we make use of density functional theory to determine the structure and relative stability of stable Mg point defects in forsterite. These models represent the ground state end-points of the hops leading to diffusion. Second, we probe the energy landscape that must by traversed by the defect during a hop. This provides us with the energy barrier that must be overcome for the hop to proceed and the structure of the transition state (the configuration with maximum energy on the minimum energy pathway between the start and the end point). Boltzmann statistics tell us how likely it is for a point defect to have enough energy at a given temperature to overcome the energy barrier while simulation of the lattice vibrations of the ground and activated state allow us to calculate the frequency at which each hop is attempted. Third, we combine information about multiple hops between different ground states using a kinetic Monte Carlo approach to access timescales long enough to observe the random walk and measure $\mathrm{Mg}$ diffusion in forsterite.

\subsection{Defect calculations using density functional theory}

All input parameters to our models of magnesium diffusion in forsterite are derived from atomic scale simulations. Specifically, we use a "planewave and pseudopotentials" approach (Payne et al., 1992), where density functional theory (DFT; Hohenberg and Kohn, 1964; Kohn and Sham, 1965) allows us to probe the energy of periodic boxes of simulated atoms. We use this to evaluate the ground state defect structures and energies, the structures and energies of the transition states, and the way atoms vibrate in these configurations. This approach allows us to calculate the hop activation energies and rates as a function of temperature and pressure. These calculations were undertaken using version 16.11 of the CASTEP code (Clark et al., 2005), which makes use of a plane wave basis for valence electrons (a cut off energy of $1000 \mathrm{eV}$ was used throughout) and pseudopotentials to describe core electrons (on-the-fly ultra soft pseudopotentials were used with $2 s, 3 p$ and $3 s, 2 s$ and $2 p, 3 s$ and $3 p$, and $1 \mathrm{~s}$ in the valence for $\mathrm{Mg}, \mathrm{O}, \mathrm{Si}$ and $\mathrm{H}$, respectively). The PBE (Perdew et al., 1996) exchange correlation functional (a revised GGA functional) was used and, Kohn-Sham wavefunctions were represented on a (4x4x4) k-point grid in reciprocal space (Monkhorst and Pack, 1976). 
We created models of $\mathrm{Mg}$ vacancies by removing an $\mathrm{Mg}^{2+}$ ion from an $\mathrm{M} 1$ or $\mathrm{M} 2$ site in a

(2x1x2) forsterite super cell. Interstitial defects were created by inserting an extra $\mathrm{Mg}^{2+}$ ion into potential interstitial sites in the structure. In both cases the cell parameters were always fixed to those of the defect free crystal to approximate the dilute limit. To account for atomic relaxation around the defects, the structure was then relaxed until the forces on all atoms were less than $0.01 \mathrm{eV} / \AA$ and an energy change between different geometric steps was less than $1 \times 10^{-5} \mathrm{eV} /$ atom. Repeating calculations with increased cutoffs changed the energy of the supercell by $<0.1 \mathrm{meV} /$ atom. $A(2 \times 1 \times 2)$ forsterite supercell was used to ensure that there was roughly $10 \AA$ between repeating vacancies in all directions, a distance we found to be sufficient to contain the important atomic relaxations. Simulation cells containing vacancies or interstitials have a net charge and so the energy calculated by CASTEP includes a defect-defect interaction term between adjacent supercells which does not reflect our desired energy of a charged defect in an infinite medium. We can approximately correct for this interaction by assuming it is the energy of a periodic array of point charges in a uniform neutralising background charge. This was done using the method of Leslie and Gillan (1985), first used for forsterite by Brodholt (1997). To use this method the relative permittivity of the cell needs to be set - we used a value of 6.2 (Weast, 1981). We repeated these calculations for a $(4 \times 2 \times 4)$ supercell containing a Mg vacancy, and the vacancy energy changed by $<0.01 \mathrm{eV}$, suggesting that our simulation cell size and energy corrections are sufficient for our needs.

Knowing the energy of defects allows us to calculate their population with assumptions about their formation reactions. The $\mathrm{Mg}$ Frenkel reaction $\left(M g_{M g}^{X} \rightarrow V_{M g}^{\prime \prime}+M g_{I}^{\bullet \bullet}\right)$ is the likely formation reaction for Mg vacancies (Dohmen and Chakraborty, 2007). This will be the source of defects in this work and the number of defects can be calculated from the knowledge of the defect energies of $\mathrm{Mg}$ vacancies and interstitials. This assumption is explored later in the text.

Once ground state structures and energies for the defects had been determined, we enumerated the possible hops (where a defect moves from location to location) and for each hop we determined the pathway and found the transition state structure and energy. We did this by using a constrained 
optimisation approach. We first determined an approximate path for the hop (for vacancy diffusion this consists of two vacancies with a $\mathrm{Mg}$ atom at a point between the vacancies, for interstitial diffusion the interstitial atom is located between stable interstitial sites). For each hop we tried multiple paths, but direct paths proved to have the lowest transition state energy in all cases. A path was defined by at least 10 images (with the $\mathrm{Mg}$ atom in different locations between the start and end point) and each image was relaxed with the migrating $\mathrm{Mg}$ fixed to the path by preventing its movement in one direction ([100] or [010] or [001]) with the fixed direction being that which has the longest distance along the path. This provides an energy profile along the path and a maximum energy point. We then searched for the transition state by moving along the path from the maximum energy point in $0.1 \AA$ steps in both directions until a maximum was found. This is the candidate transition state. While this method may not definitely find the transition state our frequency calculations (below) typically returned a single imaginary eigenvalue of the dynamical matrix, as expected for a transition state. In the few cases, which were all for interstitial diffusion, where this was not the case the candidate transition state was found by manual adjustment based on visualising the eigenvectors of the imaginary phonon frequencies until a single imaginary eigenvalue was found. It turned out that this manual adjustment changed the activation energy of the hop by $<0.01 \mathrm{eV}$ suggesting that the constrained optimisation method is highly reliable for finding activation energies even if they are in complex parts of the energy hypersurface.

We repeated the calculations described above at 0,5 and $10 \mathrm{GPa}$ and 1000,1300 and $1600 \mathrm{~K}$ by setting the simulation cell dimensions to minimise the Gibbs free energy of the defect free cell. The effect of pressure is easily accounted for by adding the PV term to the internal energy of the system. The effect of temperature requires consideration of the thermal motion of the atoms. We include this effect by making use of lattice dynamics to evaluate the phonon frequencies and then use these to evaluate the vibrational entropy of the crystal. Phonon frequencies were determined using the finite displacement method of CASTEP with finite displacements of 0.01 bohr. All lattice dynamics calculations were performed solely at the $q=(0,0,0)$ point. While this calculation at a single $q$-point 
may introduce a significant sampling error all of our calculations involve comparisons between two very similar structures - the start/end point of a diffusion step and its transition state - and so the effect of sampling errors are likely to be small but this is a limitation of the method. For lattice dynamics we tightened the convergence criteria on the forces and energy for the geometry optimisation to $0.001 \mathrm{eV} / \AA ̊$ and $1 \times 10^{-9} \mathrm{eV} /$ atom, respectively. A few end points and transition states were sampled with $0.00075 \mathrm{eV} / \AA \AA \AA$ and $5 \times 10-{ }^{10} \mathrm{eV} /$ atom cuts off and the change in free energy caused by these increased cutoffs was $<1 \mathrm{meV} /$ atom. We determined the Gibbs free energy at a wide range of temperatures and at least 5 different volumes and then the energy at each volume with the following equations:

$$
G(P, T, V)=U(V)+P V+E_{Z P}(V)-T S(T, V) \text { Equation } 1
$$

$$
E_{Z P}(V)=\sum_{k, i} \frac{1}{2} \hbar v_{k, i}(V) \text { Equation } 2
$$

$$
S(V)=-\sum_{k, i} \ln \left[1-\exp \left(-\frac{\hbar v_{k, i}(V)}{k_{B} T}\right)\right]-\frac{1}{T} \sum_{k, i} \hbar v_{k, i}(V)\left[\exp \left(\frac{\hbar v_{k, i}(V)}{k_{B} T}\right)-1\right]^{-1} \text { Equation } 3
$$

Where $U(V)$ is the internal energy and $v_{k, i}(V)$ is the frequency of the phonon with wave vector $k$ in the $i$-th band at volume $\mathrm{V}$. At the pressure and temperature of interest the appropriate volume and energy was determined by fitting $2^{\text {nd }}$ order polynomials across our volume range and minimising Equation 1. This method is quasi-harmonic as it ignores anharmonic effects beyond those caused by thermal expansion.

\subsection{From defects to diffusion}

The self-diffusion of a Mg by a vacancy mechanism can be represented by:

$$
D_{M g}^{s d-v a c}=D_{M g}^{V a c} N_{V a c} \text { Equation } 4
$$

Where $D_{M g}^{V a c}$ is the diffusion coefficient of $\mathrm{Mg}$ vacancies and $\mathrm{N}_{\mathrm{Vac}}$ is the atomic fraction of $\mathrm{Mg}$ vacancies.

As shown below, our atomic scale simulations suggest that diffusion of both interstitials and vacancies can be important for magnesium diffusion in pure forsterite. To account for this possibility 
we use the assumption that vacancies and interstitials diffuse independently of each other, which means that the total self-diffusion of $\mathrm{Mg}$ in forsterite is given by:

$$
D_{M g}^{s d}=D_{M g}^{V a c} N_{V a c}+D_{M g}^{I n t} N_{I n t} \text { Equation } 5
$$

Other diffusing species (which are not considered in this paper) would have their own term if present. For systems with simple geometry, the diffusion coefficients can be found analytically from the attempt frequency, the migration entropy, the activation energy and the crystal structure. For example, for a single hop the coefficient is given by (Poirier, 1985):

$$
D_{M g}^{V a c}=\frac{\alpha}{q} l^{2} \operatorname{vexp}\left(\frac{\Delta S_{m}}{k_{B}}\right) \exp \left(-\frac{\Delta H_{m}}{k_{B} T}\right) \text { Equation } 6
$$

where $\alpha$ is a geometric prefactor to account for the degeneracy of the hop, $q$ is a dimensionality constant ( $q=2,4$ or 6 for 1, 2 or 3D diffusion), / is the length of the hop and the two exponential terms are the migration entropy and the migration enthalpy, respectively. This approach has been used to determine diffusion coefficients in a number of minerals including $\mathrm{MgO}$, bridgmanite and postperovskite (e.g. Vocaldo et al. 1995; Ammann et al. 2010). However, forsterite diffusion involves defects moving from one site to an inequivalent site via multiple different hops and so it becomes cumbersome to attempt to develop equations of this type. Instead we seek a numerical estimate of the diffusion coefficients by implementing a kinetic Monte-Carlo (KMC; Bortz et al. (1975)) simulation of the motion of a defect in a forsterite crystal.

For our $\mathrm{KMC}$ method we need to know the concentration of defects and the rate at which each $\mathrm{Mg}$ hop can occur. As explained above the concentration of intrinsic defects (vacancies and interstitials) were determined by minimising the free energy of the Frenkel reaction at the appropriate P and T. To determine the rate of hopping we used lattice dynamics to probe the vibration of atoms around the point defects in their ground state and transition state configurations. This allows us to model the effect of temperature on point defect mobility. The rate, $k$, at which a defect hops from one location to another is given by:

$$
k=v \exp \left(\frac{\Delta S_{m}}{k_{B}}\right) \exp \left(-\frac{\Delta H_{m}}{k_{B} T}\right) \text { Equation } 7
$$


where $v$ is the attempt frequency (in $\mathrm{Hz}$ ). The activation energy term was calculated from our constrained optimisation. In order to calculate the attempt frequency and activation entropy we used Vineyard theory (Vineyard, 1957) which is based on absolute rate theory. Both of the temperaturebased factors (vibrational entropy and attempt frequency) are combined into a modified attempt frequency $\left(v^{*}\right)$ which is found from the ratio of the calculated phonon frequencies:

$$
v^{*}=\operatorname{vexp}\left(\frac{d S}{k_{B}}\right)=\frac{\prod_{j=1}^{N} v_{j}}{\prod_{j=1}^{N-1} v_{j^{\prime}}} \text { Equation } 8
$$

where $v_{j}$ are the lattice frequencies of a defect in its stable starting position and $v_{j}^{\prime}$ are the real lattice frequencies of the defect at the transition state of its hop. The latter has one imaginary frequency and so one less real frequency. Similarly to our method for the treatment of thermal expansion this theory assumes harmonic small oscillations near the saddle point and thus assumes the system operates as a harmonic oscillator. Once activation energies and modified attempt frequencies have been calculated for each hop (and at each temperature and pressure of interest), we can calculate the rate of each hop and have all the atomic scale information in hand to evaluate the absolute diffusivity of magnesium in forsterite. These single hop parameters are then fed into our KMC algorithm.

First developed to allow the efficient simulation of Ising spin systems, KMC works by simulating the time evolution of a system between a collection of states, with transitions between states governed by a set of rules that includes a probability of that transition occurring in a given amount of time. Transitions between states are selected randomly (preserving the relative probability of each transition) and a clock is advanced by an appropriate amount after the state transition has been determined. This makes it useful for simulating complex transitions with many possible motions as the properties of each transition can be calculated independently and then put collectively into a KMC algorithm. KMC has found a number of applications in extending atomic scale simulations to macroscopic behaviour, including the simulation of dislocation motion (Bulatov and Cai, 2006), chemical vapour deposition (Bagatur'yants et al., 2003) and point defect diffusion (Voter, 2007). 
For our simulations, we followed the rejection-free residence time method of Voter (2007). A brief overview of this method shall be given here, with more detail in the Supplementary Information. For each state in the system (e.g. a vacancy on $\mathrm{M} 1$ ) we enumerate all possible hops from that state and then calculate the rate of each hop $\left(k^{i}\right)$ (equation 7 using equation 8), the sum of the rates of all the hops $\left(\mathrm{k}^{\mathrm{tot}}\right)$ and the probability of each hop occurring $p^{i}=\frac{k^{i}}{k^{\text {tot }}}$. We then use the weighted probability of each hop to randomly select a hop. We also randomly select a time for that hop to occur (the escape time):

$t^{i}=-\left(\frac{1}{k_{t o t}}\right) \ln \left(r^{2}\right)$ Equation 9

Where $r^{2}$ is a random number between 0 and 1 . At each stage of the calculation the randomly selected hop moves our defect a certain distance in a certain direction and the randomly determined escape time advances the clock. Thus as this algorithm progresses, we build a list of positions of the defect as a function of time as it undergoes a random walk through the (infinite) crystal structure. We then calculate the mean-squared displacement (MSD) of our defect (using the method of Leetmaa and Skorodumova (2015) as explained in the supplementary information) as a function of time. This can then be converted to diffusion:

$\left\langle x^{2}\right\rangle=q D t$ Equation 10

Where $q$ is the dimensionality constant as above.

\section{Results}

\subsection{Defect Energies and Concentrations}

There are two sites for $\mathrm{Mg}$ vacancies in forsterite - the M1 and the M2 sites. We calculate that M1 sites are strongly favoured over M2 sites with pressure increasing the vacancy preference for $\mathrm{M} 1$ sites (Table S1). This preference for M1 over M2 vacancies agrees with previous calculations though there is some difference in the energy of this preference $(0.9-1.2 \mathrm{eV}$ in this work, $\sim 1.9 \mathrm{eV}$ with forcefield calculations (Walker et al., 2009) or $\sim 0.8 \mathrm{eV}$ previously using DFT (Brodholt, 1997)). 
We have also considered Mg interstitials. As with Walker et al. (2009) we found that the most stable position is a split interstitial at the M1 site with $2 \mathrm{Mg}$ atoms displaced from the centre of this site in opposite [010] directions (shown in Figure S1). This arrangement is very stable with alternative arrangements of the $\mathrm{Mg}$ at this site all relaxing into this one. Even placing a $\mathrm{Mg}$ atom in an $\mathrm{I}$ site causes it to relax into this split interstitial arrangement. The other stable configuration is found by placing an additional $\mathrm{Mg}$ in the 12 site. The $\mathrm{Mg}$ interstitial in the 12 site has an octahedral coordination like the $\mathrm{M} 1$ and $\mathrm{M} 2$ and is thus geometrically similar to them. At $0 \mathrm{GPa}$ the split M1 interstitial is slightly favoured over the 12 arrangement $(\sim 0.2 \mathrm{eV})$ but with increasing pressure the 12 configuration is favoured (Table S1) as the split M1 arrangement is larger than the 12 arrangement. In QM-MM embedded cluster calculations (Walker et al., 2009) the split M1 geometry was found to be favoured over an I1 interstitial geometry by $\sim 4.4 \mathrm{eV}$ but an 12 geometry was not reported. In our own forcefield calculations we were unable to stabilise an 12 arrangement as 12 arrangements always relaxed into $M 1$ arrangements. Forcefields are thus likely poor at calculating these interstitial structures.

To calculation diffusion rates the concentration of vacancies is required (Equation 5). For intrinsic diffusion we have assumed this comes from minimising the free energy of the Frenkel reaction $\left(M g_{M g}^{X} \rightarrow V_{M g}^{\prime \prime}+M g_{I}^{\bullet \bullet}\right.$. When this reaction proceeds forward the positive enthalpy and the negative configurational entropy term both increase and at some concentration this provides a minimum energy. As the Mg interstitial is able to occupy two sites solving analytically for the free energy minimum is awkward. Instead we calculate the number of different arrangements of $\mathrm{Mg}$ vacancies and defects in the crystal considering all M1, M2, I1 and 12 sites and then calculate the probability of their occurrence and thus their configurational entropy. The steps for this are given in the supplementary information but the final result is that the equilibrium concentration in the intrinsic case comes from minimising Equation 11:

$\Delta G=\Delta E \times a-T S_{\text {confa }}$ Equation 11 
where a is a reaction vector for the Frenkel reaction (between 0 and 1 ), $\Delta \mathrm{E}$ is the energy of the Frenkel reaction and $\mathrm{S}_{\text {confa }}$ is the configurational entropy after the reaction has proceeded forward by $\mathrm{a}$. The results of this minimisation are given in Table 1. Pressure strongly decreases the number of defects (by increasing the positive formation energy) whereas temperature increases the number of defects (as the configuration entropy is multiplied by $-\mathrm{T}$ ).

To test the assumption that only the Frenkel reaction is important we looked at the following intrinsic reactions that produce $\mathrm{Mg}$ defects:

R1) $M g_{M g}^{X} \rightarrow V_{M g}^{\prime \prime}+M g_{I}^{\bullet \bullet}$

R2) $M g_{M g}^{X}+O_{O}^{X} \rightarrow V_{M g}^{\prime \prime}+V_{O}^{\bullet \bullet}+M g O$

R3) $M g O \rightarrow M g_{I}^{\bullet \bullet}+O_{I}^{\prime \prime}$

R4) $2 V_{M g}^{\prime \prime}+S i_{S i}^{X}+4 M g O \rightarrow V_{S i}^{\prime \prime \prime \prime}+2 M_{M g}^{X}+M_{2} \mathrm{SiO}_{4}$

R5) $\mathrm{Si}_{S i}^{X}+4 \mathrm{MgO} \rightarrow V_{S i}^{\prime \prime \prime \prime}+2 \mathrm{Mg}_{I}^{\bullet \bullet}+\mathrm{Mg}_{2} \mathrm{SiO}_{4}$

R6) $\mathrm{Mg}_{2} \mathrm{SiO}_{4}+\mathrm{Si}_{S i}^{X}+2 \mathrm{Mg} g_{M g}^{X} \rightarrow 2 V_{M g}^{\prime \prime}+S i_{I}^{\cdots \cdots}+S i_{S i}^{X}+4 M g O$

To simulate the other defects we probed all likely sites, found the sites with the minimum enthalpy and then calculated their high temperature energy through Equation 1-3. The energy of these reactions is shown in Table S2 but all reactions have substantially higher energies than the Frenkel reaction (R1). By including these other reactions, which also compete in configurational entropy space, we change the concentration of $\mathrm{Mg}$ defects by less than $0.001 \%$ and thus these can safely by ignored and we shall only consider the Frenkel reaction R1 from now on.

\subsection{Vacancy Hops}

For Mg diffusion by vacancy hopping we found six different vacancy diffusion hops for which we calculated the geometries and energies of hopping. The hops that we have considered are shown and labelled in Figure 1 with their dimensions listed in Table S3 and described in the SI. 
The activation energies and frequencies of these hops are presented in Table 2 and the barriers to diffusion are shown in Figure 2. Notably the A hop which is directly along the [001] direction has a substantially lower activation energy than all other M1 hops. The easiest hop from an M2 site is the $\mathrm{C}$ hop back to an M1 site. These two effects combine such that vacancies will diffuse easily along the [001] direction when in a M1 site and will have difficulty escaping to an M2 site. If they do escape to an $\mathrm{M} 2$ site they will be converted quickly back to an M1 site. The weighted probability of these hops is shown in Figure 2 and an alternative representative in Figure S2 demonstrating the overwhelming dominance of the A hop.

Comparing our activation energies to published values we find that our value for the favoured A hop of $0.75 \mathrm{eV}$ is similar to literature values of 0.72 (Walker et al., 2009) and $0.62 \mathrm{eV}$ (Bejina et al., 2009). Our other hops have some variation with those found in Walker et al. (2009). To test whether this was an effect of simply using DFT as against using forcefields we recalculated our results using GULP with a TBH1 forcefield (Wright and Catlow, 1994) (Table S4, computational details in supplementary information). We find that generally DFT produces lower barriers than forcefield calculations but that the order of the hops is the same with both DFT and forcefield calculations. Crucially the activation energy of the easiest A hop (which largely controls the overall diffusion) is very similar with both methods $0.77 / 0.75 \mathrm{eV}$ which means that both DFT and forcefield calculations return a very similar diffusivity for anhydrous vacancy diffusion.

We also considered the effect of pressure on the activation energies of these vacancies. As shown in Table S5 and Table S6 going from 0 to $10 \mathrm{GPa}$ makes negligible differences to the activation energy or $v^{*}$ of any of the hops. The small differences seen are miniscule compared to the effect pressure has on the vacancy concentration as described above.

\subsection{Mg interstitial hops}

As $\mathrm{Mg}$ interstitials occupy $\mathrm{M} 1$ and $\mathrm{I} 2$ sites- the latter of which are simply shifted $\mathrm{M} 2$ sites- the relative geometry of interstitial hops are identical to those of vacancies. These hops are pictured and labelled in Figure 3 and their barriers in Figure 4 (and tabulated in Table S7) with their energies and frequencies 
in Table 2 (and more pressure derivatives listed in Table S6 and S8). The probability of any of the hops occurring is shown in Figure 4 and alternatively in Figure S3. These are again described in the supplementary information.

Interstitial hops I and J, which are between M1 and I2 sites, are the most favourable with activation energies $<0.6 \mathrm{eV}$. In part this is because in the split M1 configuration one Mg at the M1 site is already close to an 12 site. Pressure has a small effect on the attempt frequency (Table S6) but a relatively large effect on the activation energy of these hops (Table S8) with hop I becoming nearly barrierless by $10 \mathrm{GPa}$. Interstitial hops from the split M1 configuration have considerably lower attempt frequencies than the typical values between $1 \times 10^{-12}$ and $1 \times 10^{-13} \mathrm{~Hz}$ whereas hops from the 12 site show more typical attempt frequencies.

\subsection{Diffusion}

Using our KMC algorithm we can convert hops into diffusion rates. The diffusion coefficients for both vacancy and interstitial hopping are presented in Table 3 (these are listed at 5 and $10 \mathrm{GPa}$ in Table S9 and S10). Vacancy diffusion is highly anisotropic with diffusion along [001] being orders of magnitude faster than diffusion along [100] or [010]. This is an outcome of diffusion where the hop directly along [001] is $\sim 0.75 \mathrm{eV}$ easier than any other M1 hop. In the absence of any additional undiscovered hops/mechanisms this will always hold. Interstitial diffusion is much more isotropic than vacancy diffusion due to the favourability of $\mathrm{M} 1$ to 12 hops (I and J) which go in all three primary directions.

To calculate total diffusion of $\mathrm{Mg}$ in forsterite we added together the rates of $\mathrm{Mg}$ vacancy and interstitial diffusion. This assumes that Mg Frenkel pairs are not associated with each other. To test this assumption, we calculated the binding energy of this pair by running separate simulations with isolated Mg vacancies and interstitials and then calculations with them adjacent in the same unit cell and comparing the difference in enthalpy. We find that the binding energy is approximately $-1.9 \mathrm{eV}$ with a negative number indicating that bound defects are more stable than unbound defects. This is a large number but it is much smaller than the configurational energy gains of randomly scattering $\mathrm{Mg}$ 
vacancy and interstitial pairs for low concentrations. For the pairing energy to exceed this configuration entropy, the defect concentration would need to be above $1.2 \times 10^{-3}$ defects per unit cell at $1300 \mathrm{~K}$, many orders of magnitude larger than the predicted vacancy concentrations (Table 1 ). Thus the Mg vacancy and interstitial pairs are unlikely to be associated with each other and can be modelled individually here.

Figure 5 compares our pressure corrected (see supplementary information) results with some experimental measures of $\mathrm{Mg}$ self-diffusion at $0 \mathrm{GPa}$. We only plotted results for experiments buffered with $\mathrm{MgO}$ because enstatite has been observed (in one case) to increase $\mathrm{Mg}$ diffusion rates by nearly 1 order of magnitude (Jollands et al., 2020). For Chakraborty et al. 1994 we plotted the results without buffer as the $\mathrm{SiO}_{2}$ activity of these experiments is likely controlled by MgO (Jollands et al., 2020). In the [001] direction our results are very similar to those of Jollands et al. 2020. The absolute value of our results, however, is somewhat unreliable as it is largely dependent on the choice of $V_{0}^{e x p}$ and thus the pressure correction. This is shown in Figure $\mathrm{S} 4$ where a larger (-5 GPa) pressure correction was applied and we find diffusion rates in the [001] direction very similar to those of Chakraborty et al. 1994. The results presented in Figure 5 use what we consider the most reliable pressure correction. Regardless our calculated [001] diffusion lies in the experimental range. In the [100] and [010] direction our results are within the experimental scatter of Andersson et al. (1989) but not that of Jollands et al. 2020. The experimental results have considerable differences from each other. While partly this is due to self-diffusion experiments being very difficult there is another possible cause. The most likely cause of these discrepancies is the presence of different extrinsic defects across different systems. Different extrinsic defects even if they do not diffuse themselves could vary the $\mathrm{N}_{\text {vac }}$ and $\mathrm{N}_{\text {Int }}$ terms in Equation 5 and thus vary the diffusion rate. Such a variation would only have a very small effect on the experimentally determined activation energy unless the extrinsic defects were themselves produced thermally. Traditional measures of crystal purity are not adequate to accurately judge this effect as the key parameter is not so much the presence of different extrinsic defects but how these defects affect the intrinsic defects on a sub ppb level. To fully address this a 
large thermodynamic model needs to be built which is beyond the scope of this work. Another possibility is that there exists some factor of diffusion- such as an additional method of defect production- that is not replicated in this study.

Our ability to replicate the results of Jollands et al. (2020) suggests that our model for diffusion in anhydrous forsterite accurately captures its diffusion in at least one case. Critically if interstitials are not included in our model while [001] diffusion can be modelled accurately, [100] and [010] diffusion would be orders of magnitude slower than has been observed by any experiment.

We next consider the effect of pressure. Figure 6 shows our anhydrous [001] diffusion rates (with values listed in Table S11) as a function of pressure. Notably we find a larger pressure derivative for intrinsic diffusion coefficients than has been seen in the literature (Chakraborty et al., 1994, Fei et al., 2018). Our activation volumes are $6.69 \mathrm{~cm}^{3} / \mathrm{mol}$ at $1000 \mathrm{~K}, 7.51 \mathrm{~cm}^{3} / \mathrm{mol}$ at $1300 \mathrm{~K}$ and $7.84 \mathrm{~cm}^{3} / \mathrm{mol}$ at $1600 \mathrm{~K}$. The pressure dependence of diffusion is strongly controlled in our calculations by the pressure dependence of defect concentration (Table 1) with little effect of the defect mobility (Table 3). Small changes to the formation energy of the Frenkel defect can have a strong effect on this dependence. If the number of defects is held constant across pressure then the calculated activation volumes are much smaller, ranging from -0.30 to $0.15 \mathrm{~cm}^{3} / \mathrm{mol}$. These lower activation volumes are of relevance for cases where pressure does not alter the number of vacancies. For example, in an extrinsic regime (where vacancies form to charge balance impurities) the vacancy concentration is not temperature or pressure dependent and only the direct effect of pressure on vacancy mobility is important. In a real crystal with few impurities there will be a balance between the number of vacancies formed intrinsically via Frenkel pairs and the number of vacancies associated with impurities. In such a case, the effective activation volume will fall between our high and low values as is observed experimentally. 


\subsection{Anistropic intrinsic diffusion}

One of the most notable features of our results is that $\mathrm{Mg}$ diffusion can be strongly anisotropic. Figure 7 shows the anisotropy of this diffusion as a function of pressure. We find that anisotropy decreases with pressure due to the increasing importance of interstitial diffusion, which is less anisotropic, while temperature has little effect on anisotropy. At $1600 \mathrm{~K}$ and $0 \mathrm{GPa}$ (corrected) we find the ratio of diffusion in different directions [001]:[100] to be 15 and [001]:[010] to 6. Experimental measures of these ratios have produced lower results with [001]:[100] having values of $\sim 3$ (Chakraborty et al., 1994), 3.5-7 (Jollands et al., 2020) and 7-40 (Andersson, 1987), and [001]:[010] having values of 4.5 (Chakraborty et al., 1994), 1.5-3.5 (Jollands et al., 2020) and 5-13 (Andersson, 1987). These experimental measurements were all at ambient pressure. Our calculated values for diffusional anisotropy are somewhat larger than has been seen experimentally. Our pressure correction method has not been calibrated for defect production and mobility and if the pressure correction was somewhat larger our anisotropies would approach the experimental range albeit its top end. One possible way to reduce the anisotropy would be to increase the ratio of $\mathrm{Mg}$ interstitials to $\mathrm{Mg}$ vacancies which could occur in the presence of extrinsic sources of Mg interstitials or impurities that fill Mg vacancies. Alternatively, there could be some macroscopic effect that reduces diffusional anisotropy in real crystals and that we do not model.

Another issue is the order of diffusional speeds in different directions. Our calculations and some experiments (Andersson et al., 1989, Jollands et al., 2020) predict diffusion rates to be ordered $[001]>[010]>[100]$ while Chakraborty et al. (1994) measured diffusion rates to be $[001]>[100]>[010]$. We do not have a source for this discrepancy as in our model both [010] and [100] diffusion are almost entirely controlled by interstitial I and $\mathrm{J}$ hops and thus their relative rate is fixed by the geometry of the crystal and not by any variable parameter. We have no hops that could selectively increase the [100] diffusion rate that are even close to being viable. Thus the presence of evidence suggesting that diffusion along [100] can be faster than diffusion along [010] suggests some kind of atomistic or macroscopic effect that is not being modelled in our system. 
While the dependence of anisotropy on pressure is large this probably has little implication in the upper mantle. After applying pressure corrections a 0-10 GPa range in the upper mantle would be equivalent to 4-16 GPa in our pressure scales. The largest changes in anisotropy come at the lowest pressures and so across the pressure range of the upper mantle, changes in Mg diffusional anisotropy with depth will typically be up to an order of magnitude except at the coldest temperatures (1000 K) where this could reach 1.5 orders of magnitude. These changes are likely to too small to have any major effects on mantle rheology that change with depth.

\subsection{The effect of extrinsic vacancies}

512 Other substances such as iron (Chakraborty, 2010, Dohmen et al., 2007, Dohmen and Chakraborty, 2007) or water (Fei et al., 2018) that are in olivine can substantially change the diffusion rate. Without substantial changes to the diffusion mechanism there are two ways this can happen 1) through modifying the concentration of defects $\left(N_{V a c}, N_{\text {Int }}\right)$ or 2) through modifying the mobility $\left(D_{M g}^{V a c}, D_{M g}^{I n t}\right)$ of defects. All substances that modify the diffusion rate likely do the former while only some do the latter. Iron can increase the number of Mg vacancies through the following reaction (Dohmen and Chakraborty, 2007, Chakraborty, 2010):

R7) $6 \mathrm{Fe}_{\mathrm{Mg}}^{\mathrm{X}}+\mathrm{SiO}_{2}+\mathrm{O}_{2}(g) \rightarrow 2 V_{\mathrm{Mg}}^{\prime \prime}+4 \mathrm{Fe}_{\mathrm{Mg}}^{\cdot}+\mathrm{Fe}_{2} \mathrm{SiO}_{4}$

whereas water can produce $(2 H)_{M g}^{X}$ vacancies. $\mathrm{R} 7$ has been invoked as the controlling reaction in $\mathrm{Mg}$ diffusion at intermediate temperatures in what is known as the Transition Metal Extrinsic Domain (TAMED) (Chakraborty, 2010). In the Fe-Mg interdiffusion case the mobility of vacancies is also modified as they include Fe self-diffusion coefficients. In the case of water the mobility of $(2 \mathrm{H})_{\mathrm{Mg}}^{X}$ could be different to $V_{M g}^{\prime \prime}$ but likely similar. In these and other cases we expect the change in the concentrations of vacancies to generally outweigh the changes to the mobility of vacancies due to the 
small number of intrinsic defects produced by the Frenkel reaction (Table 1). Extrinsic Mg vacancy concentrations can be many orders of magnitude higher than our predicted intrinsic Mg vacancy concentration in many systems. This is seen in iron-containing olivine (Dohmen and Chakraborty, 2007) where the Mg vacancy concentration is many orders higher than predicted here due to R7. Thus the prime reason that various contaminants cause an increase in $\mathrm{Mg}$ diffusion rates is likely to be the production of more Mg vacancies. Critically Mg vacancies can be produced in this way but producing extrinsic Mg interstitials is much more difficult. This means that extrinsic defects are likely to produce a strong imbalance in the $\mathrm{Mg}$ vacancy vs $\mathrm{Mg}$ interstitial ratio.

Producing Mg vacancies in excess of Mg interstitials would cause strong changes to the anisotropy of diffusion. This effect is explored in Figure 8 where we plot how increasing the diffusion rate solely by adding $\mathrm{Mg}$ vacancies changes the anisotropy of diffusion. We are not aware of any studies on diffusional anisotropy in forsterite with large amounts of defects so instead we use our model to predict the anisotropy from the measured diffusion rates. We do this by assuming two things: 1) any change in $\mathrm{Mg}$ diffusion rate from the $\mathrm{Mg}$ self diffusion rate $\left(\mathrm{D}_{\mathrm{sd}}\right)$ in Equation 5 is due to extrinsic $\mathrm{Mg}$ vacancies and 2) that extrinsic $\mathrm{Mg}$ vacancies do not bind to any charge balancing impurities that produce them ( $D_{M g}^{V a c}$ is identical for intrinsic and extrinsic $\mathrm{Mg}$ vacancies). Then we use Equation 12: $D_{x}=D_{s d}+D_{M g}^{V a c} N_{\text {Vacex }}$ Equation 12 where $D_{x}$ is the target diffusion rate, $D_{s d}$ is determined from Equation 5 and $N_{\text {vacex }}$ is the concentration of extrinsic $\mathrm{Mg}$ vacancies that is varied until $\mathrm{D}_{\mathrm{x}}$ matches the desired value. Using this framework we explore the effects of two defective elements Fe (1-20\%) (Dohmen and Chakraborty (2017) and water (1-100 ppm) (Fei et al. 2018). At $1300 \mathrm{~K}$ we predict these defects to increase diffusional anisotropy (compared to perfect forsterite) by 2-5 times at 5-10 GPa (uncorrected). As temperature increases this effect decreases such that by $1600 \mathrm{~K}$ iron and water increase diffusional anisotropy by less than 1.2 times. Thus at the high pressures and temperatures of the upper mantle the measured experimental diffusion rates of both water and iron containing forsterite can be matched by adding in 
552

553

554

555

556

557

558

559

560

561

562

563

564

565

566

567

568

569

570

571

572

573

574

575

\section{Acknowledgments} upper mantle.

\section{Conclusions} with them.

extrinsic Mg defects without large increases in diffusional anisotropy. Thus for these compositional ranges we do not expect extrinsic vacancies to lead to significant anisotropy for Mg diffusion in the

We find that the anisotropy of $\mathrm{Mg}$ diffusion in forsterite is heavily dependent upon conditions with pressure strongly decreasing the anisotropy while temperature only weakly affects it. In the presence of extrinsic vacancies temperature strongly controls diffusional anisotropy with samples at low temperatures having potentially extremely high diffusional anisotropy (>500 times faster in the [001] direction). This has strong implications for diffusion chronometry and conductivity (and other properties dependant on $\mathrm{Mg}$ diffusion rates) which need to include corrections for pressure and impurity content alongside orientation to account for this effect.

In this work we outline a simple atomistic model which is able to replicate measured experimental diffusion rates along the [001] direction. We find that to explain experimental diffusion rates in the [100] and [010] directions interstitial diffusion is required alongside Mg diffusion.

The next step is to consider how other components could affect this diffusion such as has been seen with enstatite (Jollands et al., 2020) and with our simple extrinsic vacancy model in Figure 8. Additional components can either affect the number and balance of $\mathrm{Mg}$ vacancies and interstitials or they can affect the intrinsic diffusion of Mg vacancies and interstitials. The former effect can be considered by examining the energetics of defect forming reactions and how contaminants change these energetics- particularly through changing the configurational entropy balances- and the latter can be considered for contaminants that directly change Mg vacancies or interstitials by interacting 
Funding was provided by the National Environment Research Council as part of the Volatiles,

578

579

580

581

582

583

584

585

586

587

588

589

590

591

592

593

594

595

596

597

598

599

600

601

602

603

604

605

606

607

608

609

610

611

612

613

614

615

616

617

618

619

Geodynamics and Solid Earth Controls on the Habitable Planet research programme (NE/M000044/1)

and by National Natural Science Foundation of China (41773057). JM is highly thankful to Chinese

Academy of Sciences (CAS) for PIFI.

\section{Bibliography}

AMMANN, M. W., BRODHOLT, J. P., WOOKEY, J. \& DOBSON, D. P. 2010. First-principles constraints on diffusion in lower-mantle minerals and a weak D " layer. Nature, 465, 462-465.

ANDERSSON, K. 1987. Materietransport und Defektstrukturen in kristallinem Magnesiumorthosilicat bei höheren TemPeraturen. Technischen Universität Clausthal.

ANDERSSON, K., BORCHARDT, G., SCHERRER, S. \& WEBER, S. 1989. Self-diffusion in $\mathrm{Mg}_{2} \mathrm{SiO}_{4}$ (forsterite) at high temperature. Fresenius Zeitschrift Fur Analytische Chemie, 333, 383-385.

BAGATUR'YANTS, A. A., KORKIN, A. A., NOVOSELOV, K. P., SAVCHENKO, L. L. \& UMANSKII, S. Y. 2003. Integrated approach to atomistic simulation of film deposition processes. In: CATLOW, C. R. A. \& KOTOMIN, E. A. (eds.) Computational Materials Science. USA: IOS Press.

BEJINA, F., BLANCHARD, M., WRIGHT, K. \& PRICE, G. D. 2009. A computer simulation study of the effect of pressure on Mg diffusion in forsterite. Physics of the Earth and Planetary Interiors, 172, 13-19.

BORTZ, A. B., KALOS, M. H. \& LEBOWITZ, J. L. 1975. NEW ALGORITHM FOR MONTE-CARLO SIMULATION OF ISING SPIN SYSTEMS. Journal of Computational Physics, 17, 10-18.

BRAITHWAITE, J. S., WRIGHT, K. \& CATLOW, C. R. A. 2003. A theoretical study of the energetics and IR frequencies of hydroxyl defects in forsterite. Journal of Geophysical Research-Solid Earth, 108.

BRODHOLT, J. 1997. Ab initio calculations on point defects in forsterite (Mg2SiO4) and implications for diffusion and creep. American Mineralogist, 82, 1049-1053.

BULATOV, V. V. \& CAI, W. 2006. Computer Simulations of Dislocations, UK, Oxford University Press.

CHAKRABORTY, S. 2010. Diffusion Coefficients in Olivine, Wadsleyite and Ringwoodite. In: ZHANG, Y. X. \& CHERNIAK, D. J. (eds.) Diffusion in Minerals and Melts.

CHAKRABORTY, S., FARVER, J. R., YUND, R. A. \& RUBIE, D. C. 1994. MG TRACER DIFFUSION IN SYNTHETIC FORSTERITE AND SAN-CARLOS OLIVINE AS A FUNCTION OF P, T AND FO2. Physics and Chemistry of Minerals, 21, 489-500.

CLARK, S. J., SEGALL, M. D., PICKARD, C. J., HASNIP, P. J., PROBERT, M. J., REFSON, K. \& PAYNE, M. C. 2005. First principles methods using CASTEP. Zeitschrift fuer Kristallographie, 220, 567-570.

DOHMEN, R., BECKER, H.-W. \& CHAKRABORTY, S. 2007. Fe-Mg diffusion in olivine I: experimental determination between 700 and 1,200 degrees $C$ as a function of composition, crystal orientation and oxygen fugacity. Physics and Chemistry of Minerals, 34, 389-407.

DOHMEN, R. \& CHAKRABORTY, S. 2007. Fe-Mg diffusion in olivine II: point defect chemistry, change of diffusion mechanisms and a model for calculation of diffusion coefficients in natural olivine. Physics and Chemistry of Minerals, 34, 409-430.

FEI, H. Z., KOIZUMI, S., SAKAMOTO, N., HASHIGUCHI, M., YURIMOTO, H., MARQUARDT, K., MIYAJIMA, N. \& KATSURA, T. 2018. Mg lattice diffusion in iron-free olivine and implications to conductivity anomaly in the oceanic asthenosphere. Earth and Planetary Science Letters, 484, 204-212. 
HARTLEY, M. E., MORGAN, D. J., MACLENNAN, J., EDMONDS, M. \& THORDARSON, T. 2016. Tracking timescales of short-term precursors to large basaltic fissure eruptions through Fe-Mg diffusion in olivine. Earth and Planetary Science Letters, 439, 58-70.

JAOUL, O. 1990. MULTICOMPONENT DIFFUSION AND CREEP IN OLIVINE. Journal of Geophysical Research-Solid Earth and Planets, 95, 17631-17642.

JAOUL, O., BERTRANALVAREZ, Y., LIEBERMANN, R. C. \& PRICE, G. D. 1995. FE-MG INTERDIFFUSION IN OLIVINE UP TO 9 GPA AT T=600-900-DEGREES-C - EXPERIMENTAL-DATA AND COMPARISON WITH DEFECT CALCULATIONS. Physics of the Earth and Planetary Interiors, 89, 199-218.

JOLLANDS, M. C., ZHUKOVA, I. A., O'NEILL, H. S. \& HERMANN, J. 2020. Mg diffusion in forsterite from 1250-1600 ${ }^{\circ} \mathrm{C}$. American Mineralogist, DOI: 10.2138/am-2020-7286.

KARATO, S., JUNG, H., KATAYAMA, I. \& SKEMER, P. 2008. Geodynamic significance of seismic anisotropy of the upper mantle: New insights from laboratory studies. Annual Review of Earth and Planetary Sciences.

LEETMAA, M. \& SKORODUMOVA, N. V. 2015. Mean square displacements with error estimates from non-equidistant time-step kinetic Monte Carlo simulations. Computer Physics Communications, 191, 119-124.

LESLIE, M. \& GILLAN, M. J. 1985. THE ENERGY AND ELASTIC DIPOLE TENSOR OF DEFECTS IN IONICCRYSTALS CALCULATED BY THE SUPERCELL METHOD. Journal of Physics C-Solid State Physics, 18, 973-982.

MORIOKA, M. 1981. CATION DIFFUSION IN OLIVINE .2. NI-MG, MN-MG, MG AND CA. Geochimica Et Cosmochimica Acta, 45, 1573-1580.

OZAWA, K. 1984. OLIVINE-SPINEL GEOSPEEDOMETRY - ANALYSIS OF DIFFUSION-CONTROLLED MGFE-2+ EXCHANGE. Geochimica Et Cosmochimica Acta, 48, 2597-2611.

PANKHURST, M. J., MORGAN, D. J., THORDARSON, T. \& LOUGHLIN, S. C. 2018. Magmatic crystal records in time, space, and process, causatively linked with volcanic unrest. Earth and Planetary Science Letters, 493, 231-241.

PAYNE, M. C., TETER, M. P., ALLAN, D. C., ARIAS, T. A. \& JOANNOPOULOS, J. D. 1992. ITERATIVE MINIMIZATION TECHNIQUES FOR ABINITIO TOTAL-ENERGY CALCULATIONS - MOLECULARDYNAMICS AND CONJUGATE GRADIENTS. Reviews of Modern Physics, 64, 1045-1097.

POIRIER, J. P. 1985. Creep in Crystals, UK, Cambridge University Press.

SCHOCK, R. N., DUBA, A. G. \& SHANKLAND, T. J. 1989. ELECTRICAL-CONDUCTION IN OLIVINE. Journal of Geophysical Research-Solid Earth and Planets, 94, 5829-5839.

TENG, F. Z., DAUPHAS, N., HELZ, R. T., GAO, S. \& HUANG, S. C. 2011. Diffusion-driven magnesium and iron isotope fractionation in Hawaiian olivine. Earth and Planetary Science Letters, 308, 317324.

TILLEY, R. J. D. 1987. Defect Crystal chemistry and its applications, United States, Kluwer Academic Publishers.

VINEYARD, G. H. 1957. FREQUENCY FACTORS AND ISOTOPE EFFECTS IN SOLID STATE RATE PROCESSES. Journal of Physics and Chemistry of Solids, 3, 121-127.

VOCADLO, L., WALL, A., PARKER, S. C. \& PRICE, G. D. 1995. ABSOLUTE IONIC-DIFFUSION IN MGO COMPUTER CALCULATIONS VIA LATTICE-DYNAMICS. Physics of the Earth and Planetary Interiors, 88, 193-210.

VOTER, A. F. 2007. INTRODUCTION TO THE KINETIC MONTE CARLO METHOD. In: SICKAFUS, K. E., KOTOMIN, E. A. \& UBERUAGA, B. P. (eds.) Radiation Effects in Solids. Dordrecht: Springer.

WALKER, A. M., WOODLEY, S. M., SLATER, B. \& WRIGHT, K. 2009. A computational study of magnesium point defects and diffusion in forsterite. Physics of the Earth and Planetary Interiors, 172, 20-27.

WEAST, R. C. A., M. J. 1981. CRC Handbook of Chemistry and Physics, Boca Raton, CRC Press.

WRIGHT, K. \& CATLOW, C. R. A. 1994. A computer simulation study of (OH) defects in olivine. Physics and Chemistry of Minerals, 20, 515-518. 
670

671

672

673

674

675

676

677

\section{6}

YOSHINO, T., MATSUZAKI, T., SHATSKIY, A. \& KATSURA, T. 2009. The effect of water on the electrical conductivity of olivine aggregates and its implications for the electrical structure of the upper mantle. Earth and Planetary Science Letters, 288, 291-300.

YOSHINO, T., ZHANG, B. H., RHYMER, B., ZHAO, C. C. \& FEI, H. Z. 2017. Pressure dependence of electrical conductivity in forsterite. Journal of Geophysical Research-Solid Earth, 122, 158171. 
Muir and Walker

Mg diffusion in forsterite

678 
Figure 1:

Diagram of possible vacancy hops between M1 and M2 sites. The absolute distances of these hops are listed in Table S3. Mg atoms are brown, Si atoms are blue with their tetrahedrons highlighted, oxygen atoms are red.

Figure 2: Plot of the activation energy barriers to Mg vacancy hopping in anhydrous forsterite. The energy of a vacancy is plotted at M1 (blue) (defined as $0 \mathrm{eV}$ ) and $\mathrm{M} 2$ (green) sites and at 7 points inbetween each site with both the site and the intermediate points plotted with the same relative energy bar as shown. Many more intermediate points were used to determine the activation energy maximum than are shown here(see text for details). Hops in the [100] direction (hops B and F) are not shown but both of these hops have activation energies higher than all the hops pictured here. The black box represents a forsterite unit cell. For a sample M1 and an M2 site we have shown the main hops with a percentage likelihood of selecting this hop that was determined at $1300 \mathrm{~K}$ and 0 GPa (uncorrected).

Figure 3:

Diagram of interstitial hops between M1 and 12 sites. The absolute distances of these hops are listed in Table S7. Octahedral holes are green.

Figure 4: As Figure 2 but for interstitial hops between $M 1$ and 12 sites with the $M 1$ sites being defined as $0 \mathrm{eV}$. The layer closer to the bottom of the graph are M1 and then 12 and M1 layers alternate going up the page. These sites are much closer in energy than the $\mathrm{M} 1$ and $\mathrm{M} 2$ sites for vacancy migration. Again hops along the $[100]$ axis $(\mathrm{H}$ and $\mathrm{L})$ are not shown but are very high in energy. With this projection I and $\mathrm{I}^{*}$ and $\mathrm{J}$ and $\mathrm{J}^{*}$ hops are on top of each other (as they are only varied along the [100] direction) but we have pictured the lower energy paths (I and J respectively).

Fig 5: Plot of experimental Mg self diffusion rates in MgO-buffered forsterite at $0 \mathrm{GPa}$ alongside our predicted rates at $0 \mathrm{GPa}$ (corrected- see supplementary information) determined by fitting between our pressure corrected values (the same plot with a 5 GPa pressure correction is shown in Fig S4). Rates have been separated by diffusion direction (colour- red= [001], green= [010], blue= [100]) and by the work they come from (symbol-see below). The lines represent our own calculations. In this collection we have excluded work in olivine and work buffered by enstatite. The mark for Fei et al. (2018a) was determined by our own extrapolation of the high temperature data across different pressures, all other points were as measured in the experiment. References are Morioka et al. 1981 triangles, Jollands et al. 2020 squares, Chakraborty et al. 1994 circles, Fei et al. (2018a) cross, Andersson et al. 1987 diamonds.

Figure 6: [001] Mg diffusion rates in perfect forsterite as a function of pressure at fixed temperatures (blue $=1000 \mathrm{~K}$, green=1300 K, red=1600 K) compared to results from Chakraborty et al. (1994) and Fei et al. (2018a). Model predictions are uncorrected (solid line) or pressure corrected (dotted line). For experimental data data points are plotted and then a line is constructed using activation volumes of 
$1.1 \mathrm{~cm}^{3} / \mathrm{mol}$ for Fei et al. (1994) and $4.3 \mathrm{~cm}^{3} / \mathrm{mol}$ for Fei et al. (2018a). The results from Chakraborty et al. (1994) are those with no buffer with an $f_{O_{2}}$ of $10^{-12}$. In these results a higher activation volume ( 3.4) was determined in air. The oxygen fugacity of Fei et al. (2018a) is unknown due to the complicated presence of water.

Figure 7: Log of the ratios of C/A ([001]/[100]) (dotted lines, circles) and C/B ([001]/[010]) Mg diffusion (solid line, squares) in perfect olivine as a function of pressure at different temperatures (blue $1000 \mathrm{~K}$, green 1300, red 1600). Two pressure scales are shown, the pressure scale from DFT and one that has been corrected as per the text.

Fig 8 Comparision of anisotropy (defined as diffusion in the [001] direction/ diffusion in the [110] direction) for different diffusion rates $\left(D_{x}\right)$ in a system of self diffusion+extrinsic vacancies. Anistropy is relative to pure forsterite which is 1 . This was determined by solving Equation 13 as a function of diffusion rate. Lines are at 1000 (blue), 1300 (orange) and $1600 \mathrm{~K}$ and solid lines represent $5 \mathrm{GPa}$, dotted lines $10 \mathrm{GPa}$ (uncorrected) which correct to around 1 and $6 \mathrm{GPa}$ respectively.

The dark region represent the range of $D_{x}$ between $\mathrm{Fe}=1-20 \%$ for olivine at $0 \mathrm{GPa}, 1300 \mathrm{~K}$ and $\mathrm{fO}^{2}=10^{-7}$ Dohmen and Chakraborty (2017). The ligt region represents the range of $D_{x}$ for water ranging between 1-150 wt. ppm at $\sim 8 \mathrm{GPA}$ and $1300 \mathrm{~K}$ (Fei et al. 2018).

Table 1: Free energy of the Frenkel reaction at various pressures and temperatures and the corresponding concentration of vacancies and interstitials (in defects/unit cell) in a pure forsterite crystal where only the $\mathrm{Mg}$ Frenkel reaction forms significant defects- this concentration is for each defect type so the concentration of total defects (vacancies+interstitials) is twice this number. Also shown is the concentration of hydrous vacancies formed by water assuming water solely forms hydrous Mg vacancies $(\gamma=1)$. All pressures are uncorrected.

Table 2: Activation energy and modified attempt frequency $v^{*}$ of various hops (shown in Figure 1 and 3 with the hop distances outlined in Table S4 and S8) for hydrous and anhydrous forsterite at 0 GPa uncorrected. Hop L could not be stabilised but is very high in energy. Hops with an asterisk go in the reverse direction where this is not equivalent.

Table 3: Diffusion coefficients $\left(\mathrm{m}^{2} / \mathrm{s}\right)$ of vacancies and interstitials in three directions at $0 \mathrm{GPa}$ (uncorrected) with 5 and $10 \mathrm{GPa}$ (uncorrected) [001] diffusion coefficients also listed. For the other coefficients in [100] and [010] at 5 and 10 GPa see Table S10 and S11.

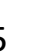
6 

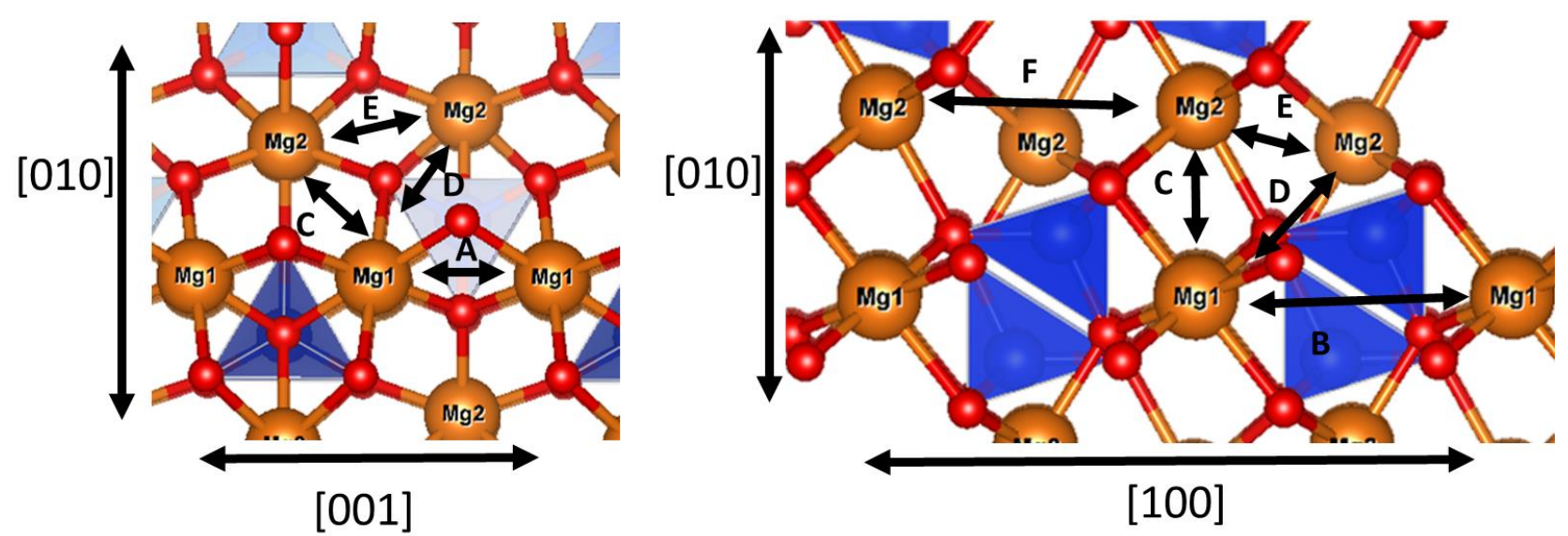

759

760 Figure 1:

761 Diagram of possible vacancy hops between M1 and M2 sites. The absolute distances of these hops

762 are listed in Table S3. Mg atoms are brown, Si atoms are blue with their tetrahedrons highlighted,

763 oxygen atoms are red. 


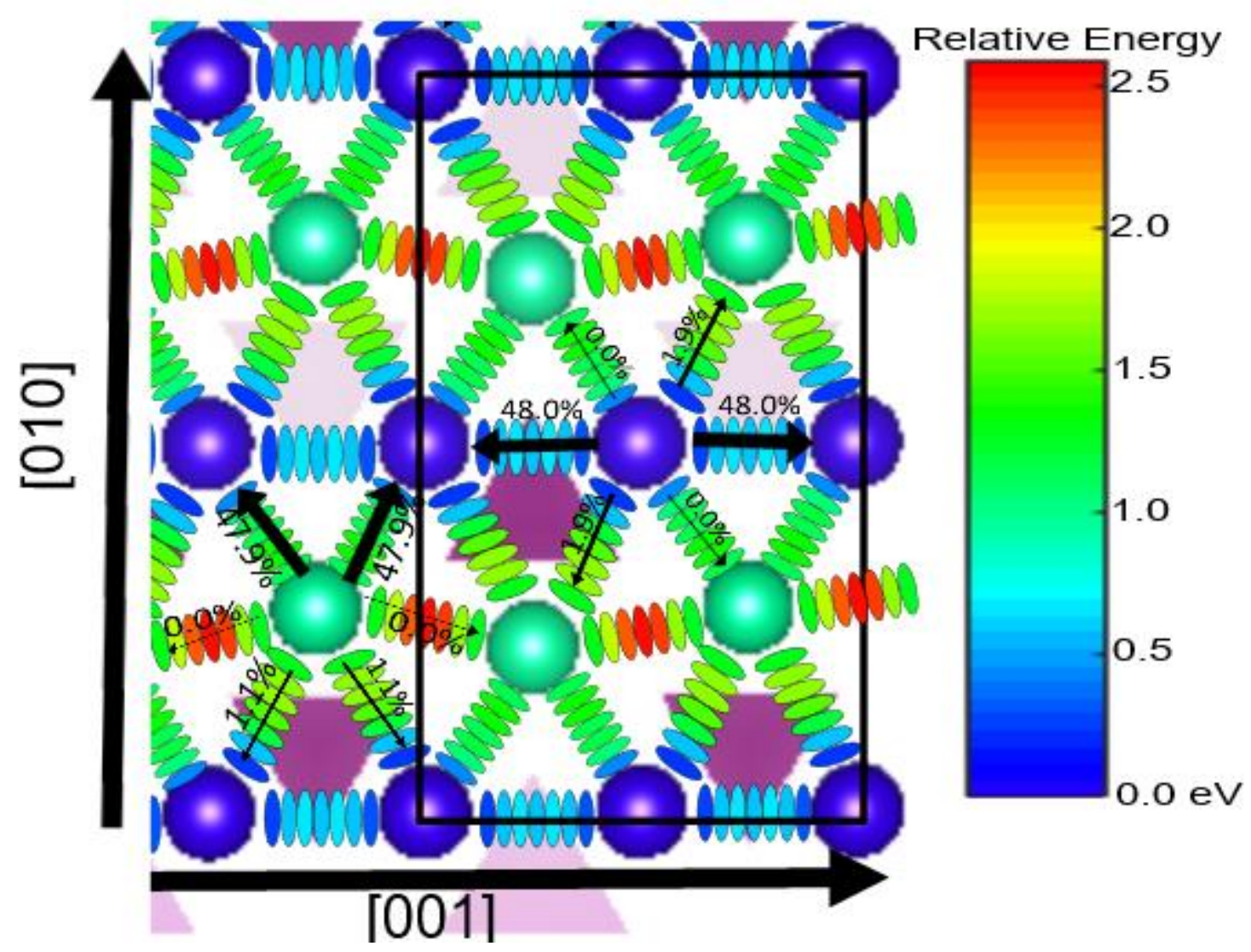

Figure 2: Plot of the activation energy barriers to Mg vacancy hopping in anhydrous forsterite. The energy of a vacancy is plotted at M1 (blue) (defined as $0 \mathrm{eV}$ ) and M2 (green) sites and at 7 points inbetween each site with both the site and the intermediate points plotted with the same relative energy bar as shown. Many more intermediate points were used to determine the activation energy maximum than are shown here(see text for details). Hops in the [100] direction (hops B and F) are not shown but both of these hops have activation energies higher than all the hops pictured here. The black box represents a forsterite unit cell. For a sample M1 and an M2 site we have shown the main hops with a percentage likelihood of selecting this hop that was determined at $1300 \mathrm{~K}$ and 0 GPa (uncorrected). 

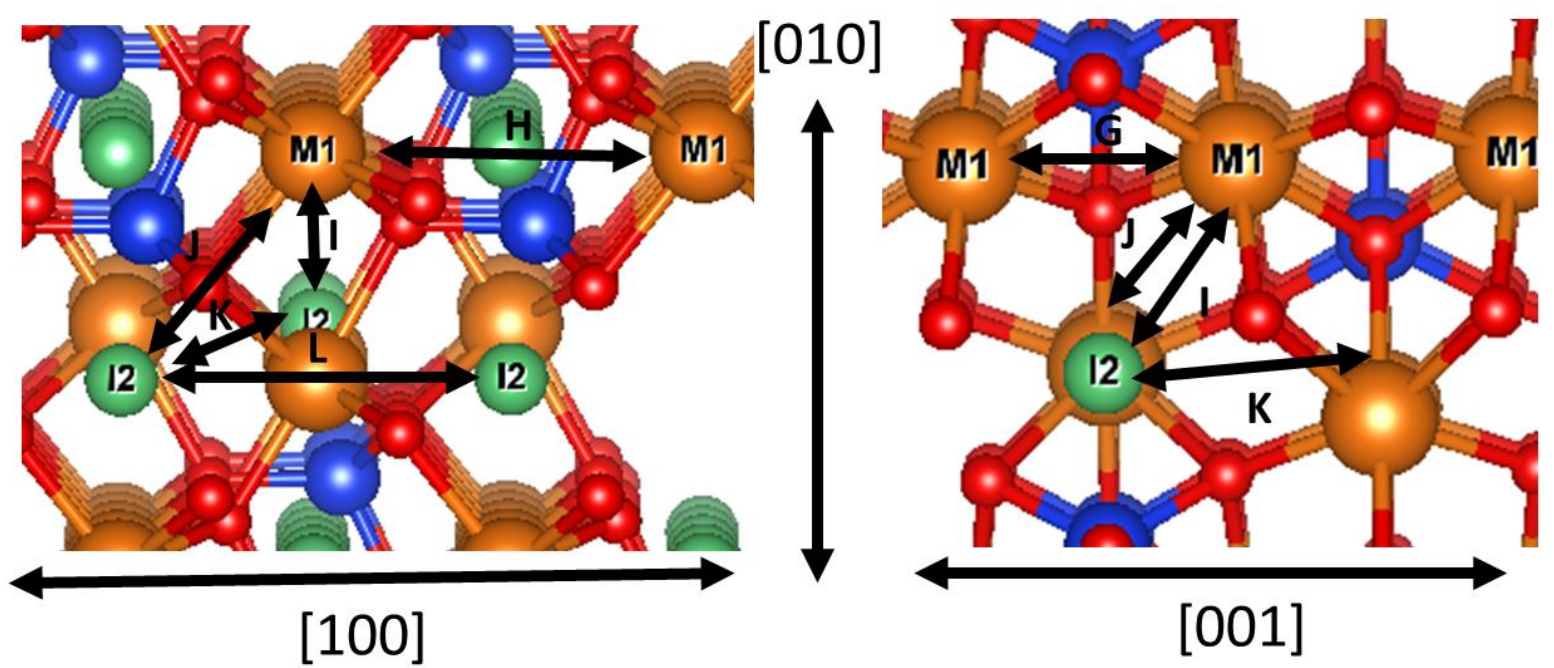

777

778

Figure 3:

779 Diagram of interstitial hops between M1 and 12 sites. The absolute distances of these hops are 780 listed in Table S7. Octahedral holes are green. 


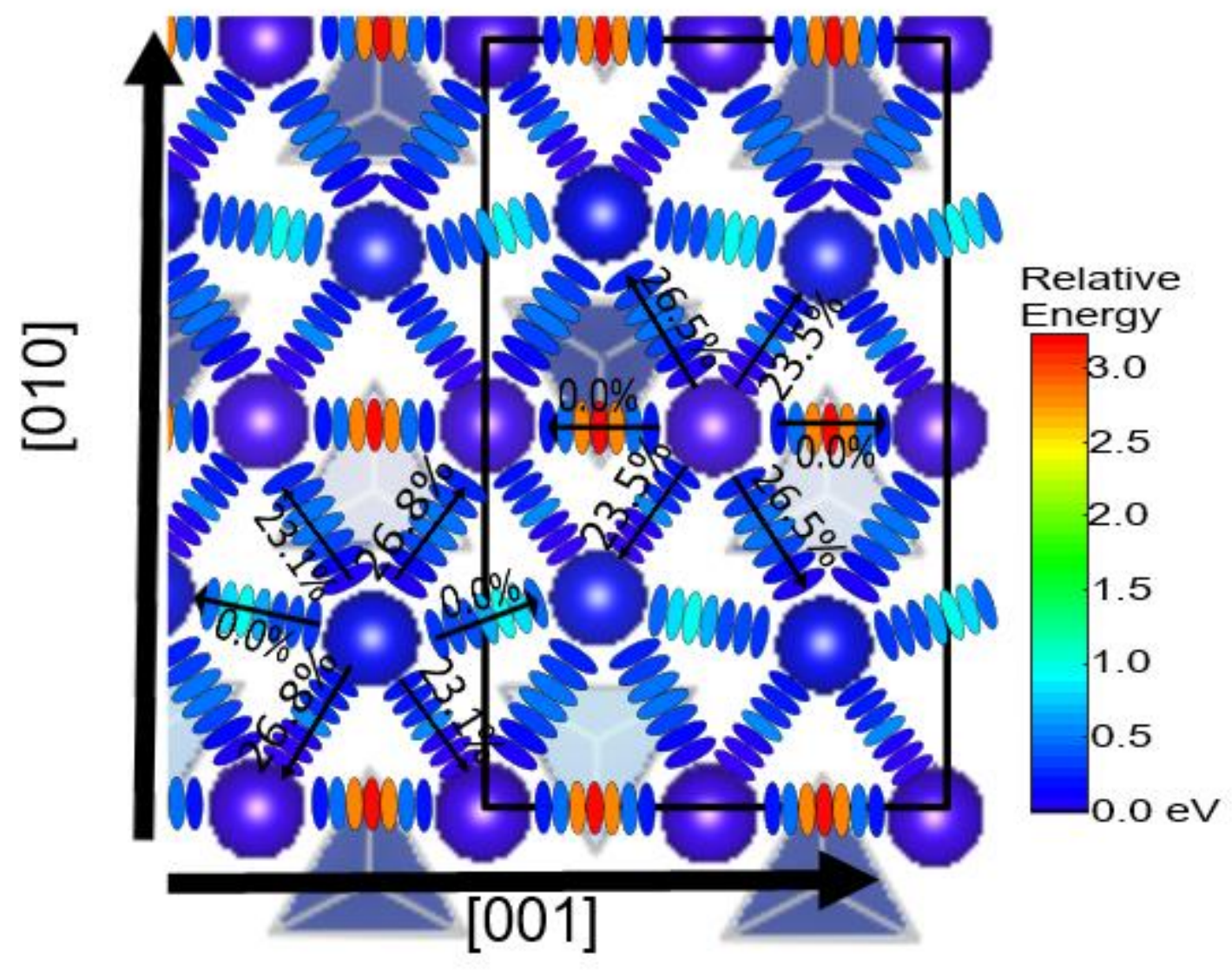

Figure 4: As Figure 2 but for interstitial hops between $M 1$ and 12 sites with the $M 1$ sites being defined as $0 \mathrm{eV}$. The layer closer to the bottom of the graph are $\mathrm{M} 1$ and then 12 and $\mathrm{M} 1$ layers alternate going up the page. These sites are much closer in energy than the $\mathrm{M} 1$ and $\mathrm{M} 2$ sites for vacancy migration. Again hops along the $[100]$ axis $(\mathrm{H}$ and $\mathrm{L})$ are not shown but are very high in energy. With this projection I and I* and $\mathrm{J}$ and $\mathrm{J}^{*}$ hops are on top of each other (as they are only varied along the [100] direction) but we have pictured the lower energy paths (I and J respectively). 


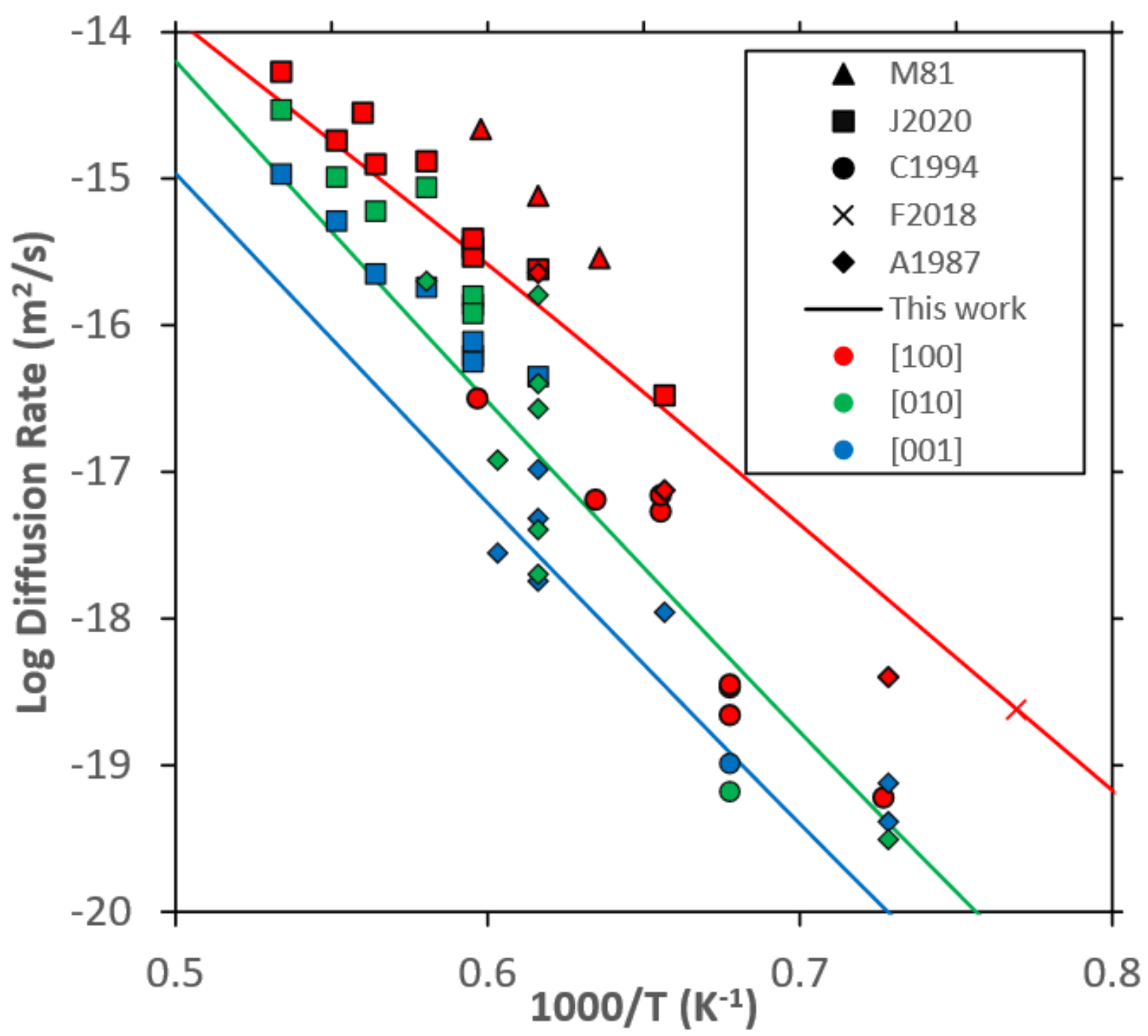

791

792

793

794

795

796

797

798

799

800

801

802

Fig 5: Plot of experimental Mg self diffusion rates in MgO-buffered forsterite at $0 \mathrm{GPa}$ alongside our predicted rates at $0 \mathrm{GPa}$ (corrected- see supplementary information) determined by fitting between our pressure corrected values (the same plot with a 5 GPa pressure correction is shown in Fig S4). Rates have been separated by diffusion direction (colour- red= [001], green= [010], blue= [100]) and by the work they come from (symbol-see below). The lines represent our own calculations. In this collection we have excluded work in olivine and work buffered by enstatite. The mark for Fei et al. (2018a) was determined by our own extrapolation of the high temperature data across different pressures, all other points were as measured in the experiment. References are Morioka et al. 1981 triangles, Jollands et al. 2020 squares, Chakraborty et al. 1994 circles, Fei et al. (2018a) cross, Andersson et al. 1987 diamonds. 


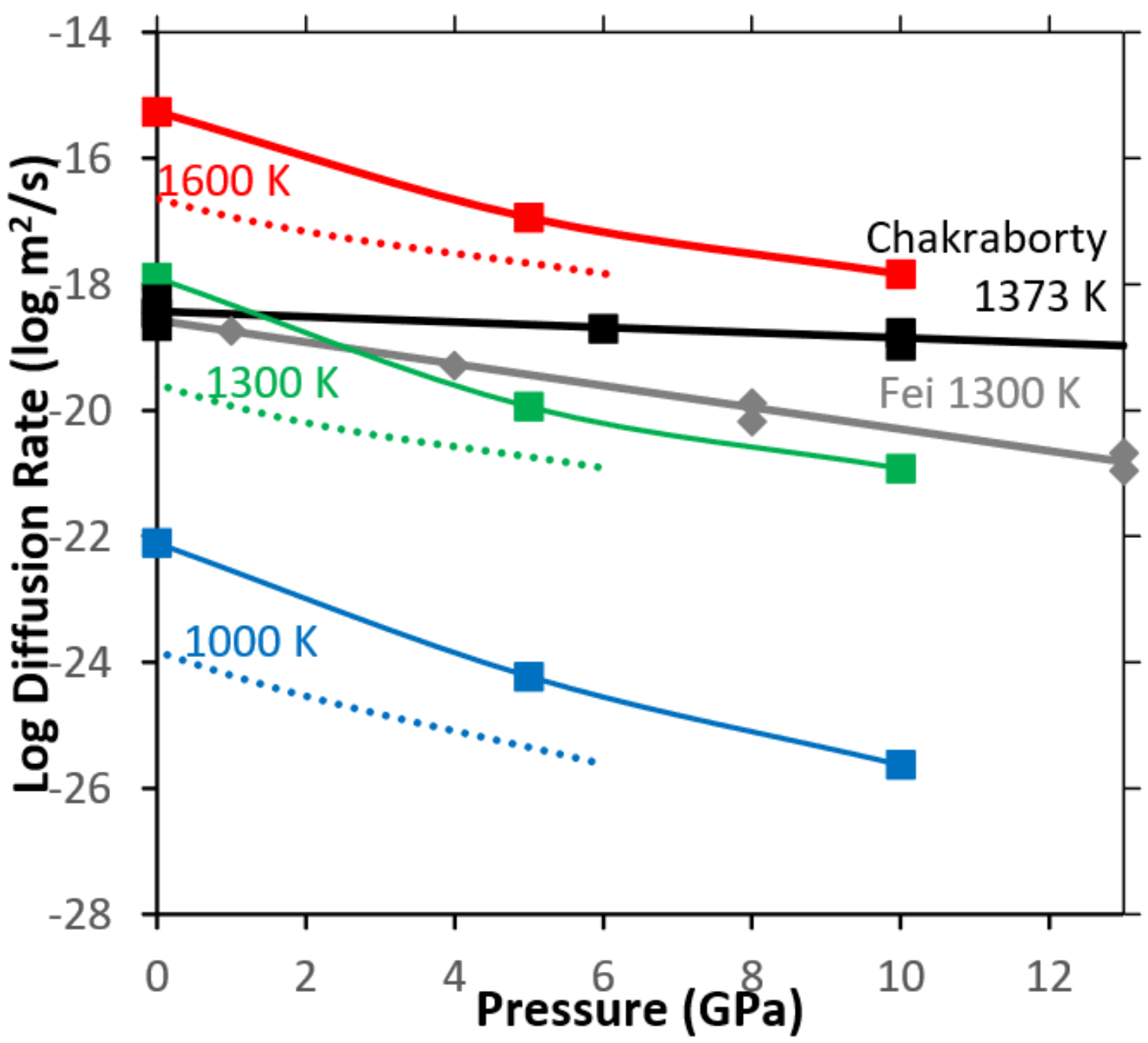

804

Figure 6: [001] Mg diffusion rates in perfect forsterite as a function of pressure at fixed temperatures (blue $=1000 \mathrm{~K}$, green $=1300 \mathrm{~K}$, red=1600 K) compared to results from Chakraborty et al. (1994) and Fei et al. (2018a). Model predictions are uncorrected (solid line) or pressure corrected (dotted line). For experimental data data points are plotted and then a line is constructed using activation volumes of $1.1 \mathrm{~cm}^{3} / \mathrm{mol}$ for Fei et al. (1994) and $4.3 \mathrm{~cm}^{3} / \mathrm{mol}$ for Fei et al. (2018a). The results from Chakraborty et al. (1994) are those with no buffer with an $f_{\mathrm{O}_{2}}$ of $10^{-12}$. In these results a higher activation volume ( 3.4) was determined in air. The oxygen fugacity of Fei et al. (2018a) is unknown due to the complicated presence of water. 


\section{Corrected Pressure (GPa)}

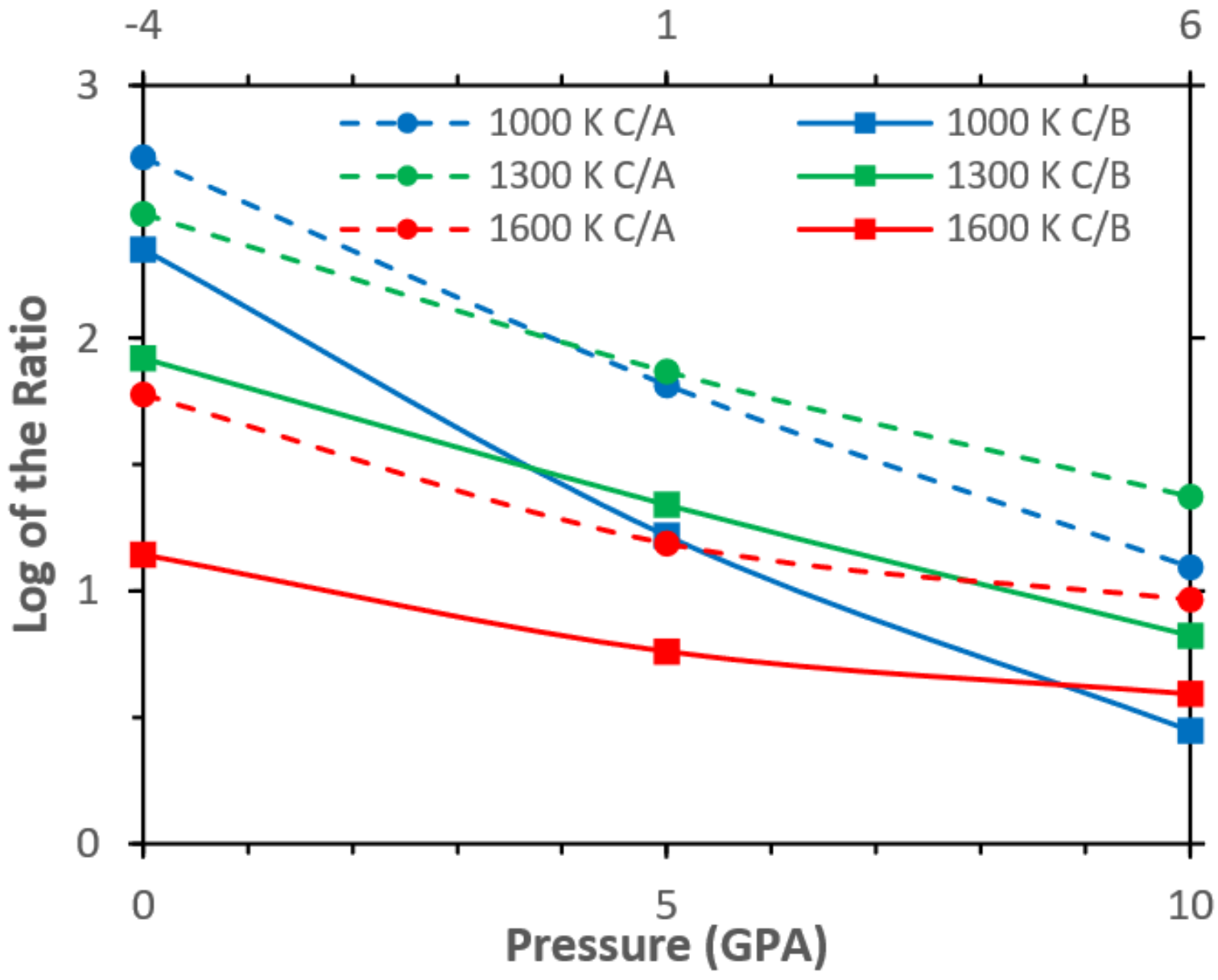

818 Figure 7: Log of the ratios of C/A ([001]/[100]) (dotted lines, circles) and C/B ([001]/[010]) Mg 819 diffusion (solid line, squares) in perfect olivine as a function of pressure at different temperatures 820 (blue $1000 \mathrm{~K}$, green 1300, red 1600). Two pressure scales are shown, the pressure scale from DFT and one that has been corrected as per the text. 


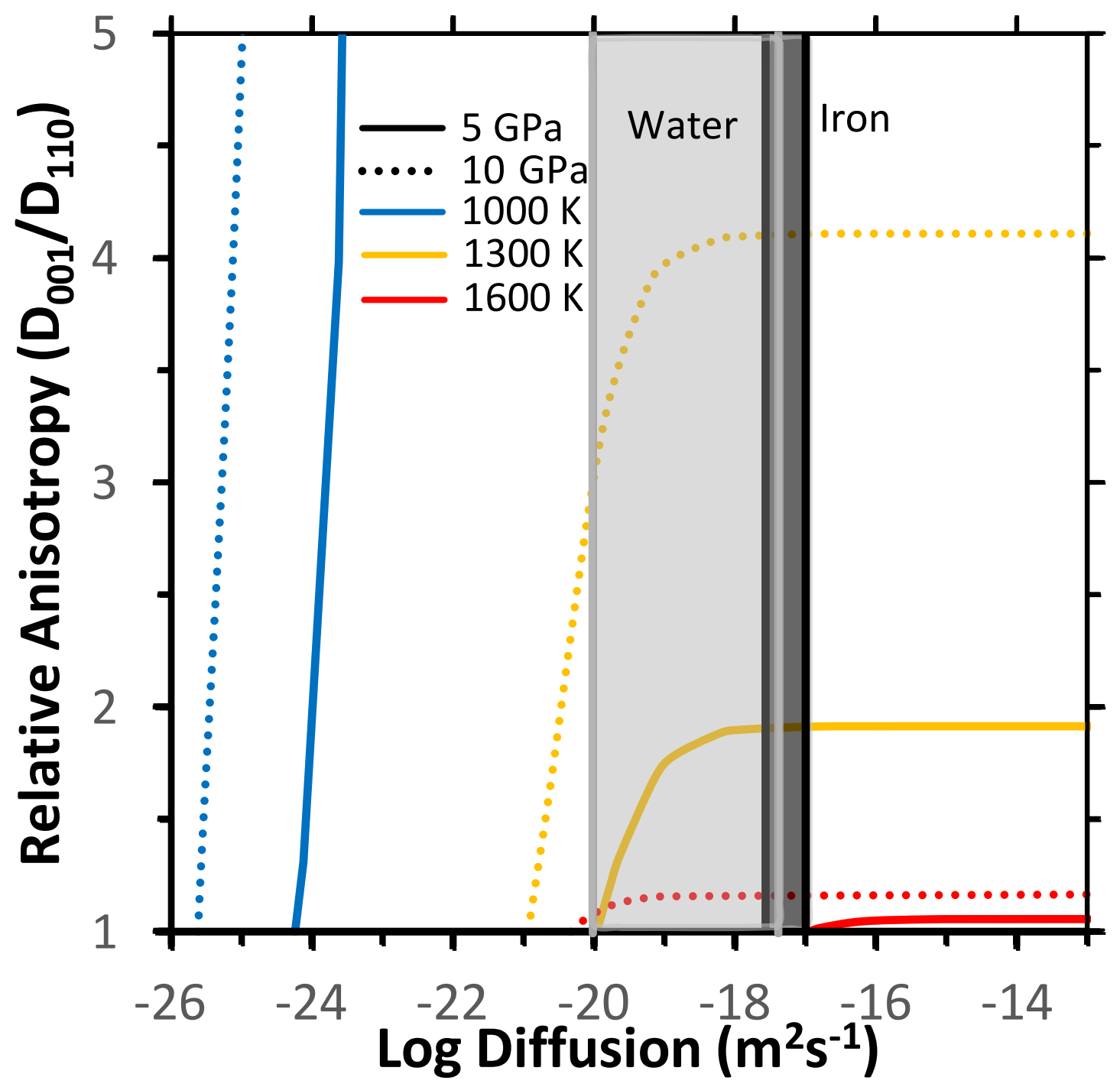

Fig 8 Comparision of anisotropy (defined as diffusion in the [001] direction/ diffusion in the [110] direction) for different diffusion rates $\left(D_{x}\right)$ in a system of self diffusion+extrinsic vacancies. Anistropy is relative to pure forsterite which is 1 . This was determined by solving Equation 13 as a function of diffusion rate. Lines are at 1000 (blue), 1300 (orange) and $1600 \mathrm{~K}$ and solid lines represent $5 \mathrm{GPa}$, dotted lines $10 \mathrm{GPa}$ (uncorrected) which correct to around 1 and $6 \mathrm{GPa}$ respectively.

830 The dark region represent the range of $D_{x}$ between $\mathrm{Fe}=1-20 \%$ for olivine at $0 \mathrm{GPa}, 1300 \mathrm{~K}$ and $\mathrm{fO}^{2}=10^{-7}$ Dohmen and Chakraborty (2017). The ligt region represents the range of $D_{x}$ for water ranging between 1-150 wt. ppm at $\sim 8$ GPA and $1300 \mathrm{~K}$ (Fei et al. 2018). 


\begin{tabular}{|r|r|r|r|}
\hline & $0 \mathrm{GPa}$ & 5 & 10 \\
\hline & \multicolumn{3}{|c|}{ Formation Energy (eV) } \\
\hline $0 \mathrm{~K}$ & 5.65 & 6.43 & 6.54 \\
\hline 1000 & 4.94 & 5.96 & 6.37 \\
\hline 1300 & 4.73 & 5.78 & 6.27 \\
\hline 1600 & 4.52 & 5.60 & 6.13 \\
\hline & \multicolumn{3}{|c|}{ Vacancy Concentration } \\
\hline 1000 & $4.13 \times 10^{-13}$ & $3.06 \times 10^{-15}$ & $9.23 \times 10^{-17}$ \\
\hline 1300 & $8.51 \times 10^{-10}$ & $8.81 \times 10^{-12}$ & $9.37 \times 10^{-13}$ \\
\hline 1600 & $9.59 \times 10^{-08}$ & $2.14 \times 10^{-09}$ & $2.89 \times 10^{-10}$ \\
\hline
\end{tabular}

Table 1: Free energy of the Frenkel reaction at various pressures and temperatures and the corresponding concentration of vacancies and interstitials (in defects/unit cell) in a pure forsterite crystal where only the Mg Frenkel reaction forms significant defects- this concentration is for each defect type so the concentration of total defects (vacancies+interstitials) is twice this number. Also shown is the concentration of hydrous vacancies formed by water assuming water solely forms hydrous $\mathrm{Mg}$ vacancies $(\gamma=1)$. All pressures are uncorrected. 


\begin{tabular}{|c|c|c|c|c|c|}
\hline & \multicolumn{2}{|c|}{ Anhydrous Vacancy } & \multicolumn{2}{|c|}{$\begin{array}{l}\text { Anhydrous } \\
\text { Interstitial }\end{array}$} \\
\hline & & $\begin{array}{l}E_{a} \\
(\mathrm{eV})\end{array}$ & $v^{*}(\mathrm{~Hz})$ & $\begin{array}{l}E_{a} \\
(e V)\end{array}$ & $v^{*}(\mathrm{~Hz})$ \\
\hline \multicolumn{6}{|c|}{ Hops from M1 Site } \\
\hline$A / G$ & M1-M1 & 0.75 & $1.01 \times 10^{13}$ & 3.22 & $9.11 \times 10^{08}$ \\
\hline $\mathrm{B} / \mathrm{H}$ & M1-M1 & 4.12 & $3.71 \times 10^{15}$ & 3.16 & $1.22 \times 10^{10}$ \\
\hline$C / I$ & $\mathrm{M} 1-\mathrm{M} 2 / 12$ & 1.45 & $2.37 \times 10^{14}$ & 0.59 & $3.01 \times 10^{09}$ \\
\hline |* & M1-12 & & & 1.35 & $2.38 \times 10^{08}$ \\
\hline$D / J$ & $\mathrm{M} 1-\mathrm{M} 2 / 12$ & 1.91 & $4.39 \times 10^{14}$ & 0.56 & $2.02 \times 10^{09}$ \\
\hline $\mathrm{D}^{*} / \mathrm{J} *$ & $\mathrm{M} 1-\mathrm{M} 2 / 12$ & 1.91 & $4.39 \times 10^{14}$ & 1.29 & $2.80 \times 10^{09}$ \\
\hline \multicolumn{6}{|c|}{ Hops From M2/12 Site } \\
\hline $\mathrm{C} / \mathrm{l}$ & $\mathrm{M} 2 / \mathrm{I2}-\mathrm{M} 1$ & 0.45 & $1.15 \times 10^{14}$ & 0.39 & $1.41 \times 10^{13}$ \\
\hline I* & M1-12 & & & 1.15 & $1.11 \times 10^{12}$ \\
\hline$D / J$ & $\mathrm{M} 2 / 12-\mathrm{M} 1$ & 1.00 & $2.13 \times 10^{14}$ & 0.36 & $9.46 \times 10^{12}$ \\
\hline $\mathrm{D*} / \mathrm{J} *$ & $\mathrm{M} 2 / 12-\mathrm{M} 1$ & 1.00 & $2.13 \times 10^{14}$ & 1.09 & $1.31 \times 10^{13}$ \\
\hline$E / K$ & $\mathrm{M} 2 / \mathrm{I2}-\mathrm{M} 2 / \mathrm{I} 2$ & 1.65 & $4.27 \times 10^{14}$ & 1.08 & $5.53 \times 10^{12}$ \\
\hline $\mathrm{F} / \mathrm{L}$ & $\mathrm{M} 2 / \mathrm{I} 2-\mathrm{M} 2 / \mathrm{I} 2$ & 2.82 & $2.31 \times 10^{15}$ & N/A & $\mathrm{N} / \mathrm{A}$ \\
\hline
\end{tabular}

846 Table 2: Activation energy and modified attempt frequency $\mathrm{v}^{*}$ of various hops (shown in Figure 1 847 and 3 with the hop distances outlined in Table S4 and S8) for hydrous and anhydrous forsterite at 0 GPa uncorrected. Hop L could not be stabilised but is very high in energy. Hops with an asterisk go in the reverse direction where this is not equivalent. 


\begin{tabular}{|l|l|l|l|l|l|r|}
\hline \multicolumn{2}{|c|}{} & {$[100]$} & {$[010]$} & {$[001]$} & {$[001] 5 \mathrm{GPa}$} & {$[001] 10 \mathrm{GPa}$} \\
\hline \multirow{4}{*}{$\begin{array}{l}\text { Anhydrous } \\
\text { vacancy }\end{array}$} & $1000 \mathrm{~K}$ & $1.58 \times 10^{-14}$ & $6.61 \times 10^{-14}$ & $1.91 \times 10^{-10}$ & $1.88 \times 10^{-10}$ & $1.88 \times 10^{-10}$ \\
\cline { 2 - 7 } & 1300 & $3.37 \times 10^{-12}$ & $1.42 \times 10^{-11}$ & $1.48 \times 10^{-09}$ & $1.27 \times 10^{-09}$ & $1.19 \times 10^{-09}$ \\
\cline { 2 - 7 } & 1600 & $9.15 \times 10^{-11}$ & $4.02 \times 10^{-10}$ & $5.71 \times 10^{-09}$ & $5.30 \times 10^{-09}$ & $4.93 \times 10^{-09}$ \\
\hline \multirow{3}{*}{ Interstitial } & 1000 & $3.47 \times 10^{-13}$ & $7.58 \times 10-13$ & $2.65 \times 10^{-13}$ & $6.13 \times 10^{-12}$ & $6.66 \times 10^{-11}$ \\
\cline { 2 - 7 } & 1300 & $1.39 \times 10^{-12}$ & $3.62 \times 10-12$ & $1.18 \times 10^{-12}$ & $1.39 \times 10^{-11}$ & $8.44 \times 10^{-11}$ \\
\cline { 2 - 7 } & 1600 & $3.83 \times 10^{-12}$ & $7.86 \times 10-12$ & $3.96 \times 10^{-12}$ & $2.46 \times 10^{-11}$ & $1.01 \times 10^{-10}$ \\
\hline
\end{tabular}

